\title{
Density changes of aerosol particles as a result of chemical reaction
}

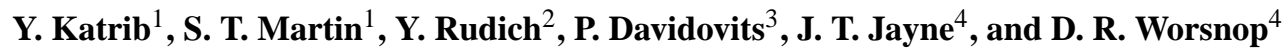 \\ ${ }^{1}$ Division of Engineering and Applied Sciences, Harvard University, Cambridge, MA 02138, USA \\ ${ }^{2}$ Department of Environmental Sciences, Weizmann Institute, Rehovot 76100, Israel \\ ${ }^{3}$ Chemistry Department, Boston College, Chestnut Hill, MA 02467, USA \\ ${ }^{4}$ Aerodyne Research, Inc., Billerica, MA 08121, USA
}

Received: 20 September 2004 - Published in Atmos. Chem. Phys. Discuss.: 8 October 2004

Revised: 13 December 2004 - Accepted: 7 January 2005 - Published: 3 February 2005

\begin{abstract}
This paper introduces the capability to study simultaneously changes in the density, the chemical composition, the mobility diameter, the aerodynamic diameter, and the layer thickness of multi-layered aerosol particles as they are being altered by heterogeneous chemical reactions. A vaporization-condensation method is used to generate aerosol particles composed of oleic acid outer layers of 2 to $30 \mathrm{~nm}$ on 101-nm polystyrene latex cores. The layer density is modified by reaction of oleic acid with ozone for variable exposure times. For increasing ozone exposure, the mobility diameter decreases while the vacuum aerodynamic diameter increases, which, for spherical particles, implies that particle density increases. The aerosol particles are confirmed as spherical based upon the small divergence of the particle beam in the aerosol mass spectrometer. The particle and layer densities are calculated by two independent methods, namely one based on the measured aerodynamic and mobility diameters and the other based on the measured mobility diameter and particle mass. The uncertainty estimates for density calculated by the second method are two to three times greater than those of the first method. Both methods indicate that the layer density increases from 0.89 to $1.12 \mathrm{~g} \cdot \mathrm{cm}^{-3}$ with increasing ozone exposure. Aerosol mass spectrometry shows that, concomitant with the increase in the layer density, the oxygen content of the reacted layer increases. Even after all of the oleic acid has reacted, the layer density and the oxygen content continue to increase slowly with prolonged ozone exposure, a finding which indicates continued chemical reactions of the organic products either with ozone or with themselves. The results of this paper provide new insights into the complex changes occurring for atmospheric particles during the aging processes caused by gas-phase oxidants.
\end{abstract}

\section{Introduction}

The density $\left(\rho_{p}\right)$ of an aerosol particle is a physical property of great importance for the prediction of particle mechanics and thus aerosol life cycles, both in the atmosphere and in the human respiratory system (Seinfeld and Pandis, 1998). The density, combined with the dynamic shape factor $(\chi)$, relates the aerodynamic diameter $\left(d_{a}\right)$ of a particle to its electric mobility diameter $\left(d_{m}\right)$ (Hinds, 1999; Baron and Willeke, 2001). The dynamic shape factor accounts for the effect of nonsphericity on the particle drag force. Furthermore, the density indirectly affects the optical properties of particles because the refractive index typically increases monotonically with the density.

Early determinations of density from measurements of the mass $\left(m_{p}\right)$ and the mobility diameter of spherical particles were made using a Millikan cell (Fuchs, 1964). More recently, Lipowicz (1988) employed a Millikan cell to determine the effective density $\left(\rho_{e}\right)$ of cigarette smoke particles. The effective density is an alternative when an experiment is not capable of separating $\rho_{p}$ and $\chi$. In this case, $\rho_{e}=f\left(\rho_{p}\right.$, $\chi)$, which can be calculated from the measurement of any two of $d_{a}, d_{m}$, or $m_{p}$ (Kelly and McMurry, 1992). Ristimaki et al. (2002) obtained the effective density from measurements of $d_{m}$ with a scanning mobility particle sizer and of $d_{a}$ with an electrical low pressure impactor. McMurry et al. (2002) determined the density of spherical liquid particles by first selecting particles of specific $d_{m}$ using an electrostatic classifier and subsequently measuring $m_{p}$ via an aerosol particle mass analyzer (Ehara et al., 1996). Hand and Kreidenweis (2002) calculated the effective density using a differential mobility analyzer to measure $d_{m}$ and an aerodynamic particle sizer to measure $d_{a}$.

Correspondence to: S. T. Martin

(scot_martin@harvard.edu)

(C) 2005 Author(s). This work is licensed under a Creative Commons License. 


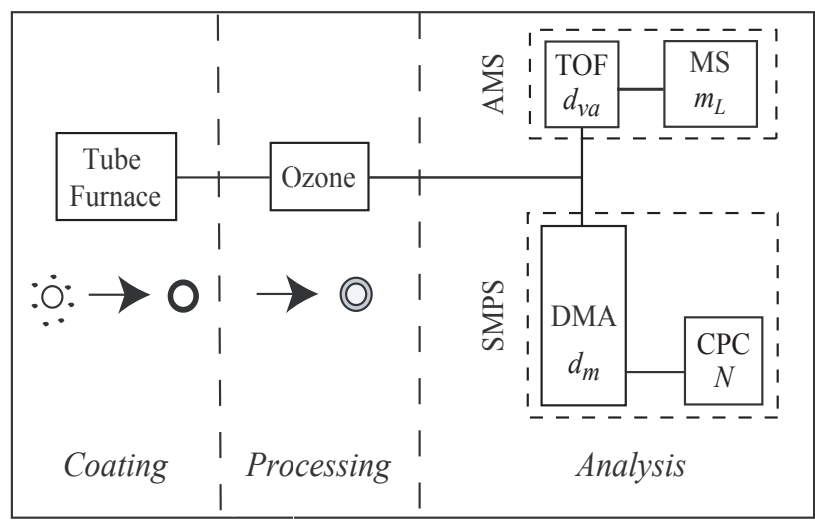

Fig. 1. Experimental apparatus for generating, processing, and analyzing coated particles. Key: TOF, time-of-flight; MS, mass spectrometer; DMA, differential mobility analyzer; CPC, condensation particle counter; AMS, aerosol mass spectrometer; SMPS, scanning mobility particle sizer. Symbols $d_{m}, d_{v a}, m_{L}$, and $N$ are defined in the text.

In comparison to these earlier reports for determining particle density, the experimental setup introduced in this paper has several significant innovations:

1. All three quantities $d_{a}, d_{m}$, and $m_{p}$ are simultaneously measured. Two independent methods of determining particle density are, therefore, possible.

2. A polystyrene latex (PSL) core serves to maintain a spherical shape for particles coated with oleic acid. Density, instead of effective density, is therefore measured. A spherical shape is confirmed by measuring the divergence of the particle beam in the AMS.

3. Particle density is systematically varied by controlled heterogeneous chemistry. Namely, ozone reacts with thin outer layers of oleic acid on the PSL core particles (Katrib et al., 2004).

The reaction of oleic acid with ozone has recently been investigated intensively (Morris et al., 2002; Moise and Rudich, 2002; Smith et al., 2002, 2003; Ziemann 2003; Thornberry and Abbatt, 2004; Katrib et al., 2004; Hearn and Smith, 2004; Asad et al., 2004; Broekhuizen et al., 2005 ${ }^{1}$ ). The experimental approach described in the current paper allows for detailed observations of the physical and chemical changes that are caused by aerosol heterogeneous chemistry.

\section{Experimental}

A scanning mobility particle sizer (SMPS) and an aerosol mass spectrometer (AMS) are employed for parallel on-line

\footnotetext{
${ }^{1}$ Broekhuizen, K. E., Thornberry, T., Kumar, P. P., and Abbatt, J. P. D.: Formation of cloud condensation nuclei by oxidative processing: unsaturated fatty acids, in press, 2005.
}

measurements of the mobility diameter, the vacuum aerodynamic diameter, the mass, and the chemical make-up of laboratory-generated aerosol particles. A detailed description of the experimental setup and protocol is provided in Katrib et al. (2004). Briefly, an aerosol composed of polystyrene latex (PSL) particles is externally mixed with an aerosol composed of oleic acid particles. The combined aerosol passes through a tube furnace having a linear hot-to-cool temperature gradient $\left(78\right.$ to $\left.25^{\circ} \mathrm{C}\right)$. The oleic acid particles vaporize in the hot region, and the vapor subsequently condenses in the cool regions onto the surfaces of the PSL particles (Fig. 1). The apparatus generates 101-nm PSL particles coated with oleic acid layers varying from 2 to $30 \mathrm{~nm}$ thickness in a reproducible and controlled manner. At the exit of the tube furnace, the coated aerosol particles are exposed to ozone of variable concentration ( 1 to $30 \mathrm{ppmV} ; 2.5 \times 10^{13}$ to $7.4 \times 10^{14}$ molec cm$^{-3}$ ) in 1 atm of $98 \% \mathrm{~N}_{2}$ and $2 \% \mathrm{O}_{2}$ for $3 \mathrm{~s}$ at a relative humidity under $1 \%$ at $298 \mathrm{~K}$. The reaction of oleic acid with $\mathrm{O}_{3}$ is employed to increase the density of the coating and to reduce the geometric diameter of the particles.

Particle shape is interrogated through measurement of the divergence of the particle beam (Sect. 2.1). Particle mobility diameter $\left(d_{m}\right)$, vacuum aerodynamic diameter $\left(d_{v a}\right)$, aerosol layer mass $\left(m_{L}\right)$, and particle number concentration $(N)$ are measured in parallel by an SMPS/AMS setup (Fig. 1) (Sects. 2.2-2.5). These primary measurements are employed to calculate particle layer mass $\left(\bar{m}_{L}\right)$, layer thickness $(L)$, particle density $\left(\rho_{p}\right)$, and layer density $\left(\rho_{L}\right)$ (Sects. 2.6-2.8). The relationships among these quantities are summarized in Tables 1 and 2. The uncertainties of the measured and calculated quantities are summarized in Table 3 .

\subsection{Interrogation of particle shape}

The divergence of a particle beam $(\Omega)$ in an aerodynamic lens similar to the one installed at the inlet of the AMS is discussed by Liu et al. (1995a, b). The divergence, which is determined in the nozzle expansion by the greater of Brownian motion or the aerodynamic lift force, depends on particle shape. A spherical particle, which provides the reference value for the drag force, has zero lift force, and, consequently, the beam divergence (caused by Brownian motion) is small.

The beam divergence inside the AMS is determined via analysis of the lateral beam profile, which is obtained by stepping a wire of $0.3 \mathrm{~mm}$ diameter across the particle beam. The solid angle of a cone having a base of radius $r$ and a height $h$ is given by $\Omega=2 \pi(1-\cos \theta)$ where $\theta=\tan ^{-1}(r / h)$. In the AMS, the distance from the expansion nozzle to the flash vaporizer is $0.45 \mathrm{~m}$.

Whereas the beam divergence is a response to the lift force, we are instead in need of the drag force for many of the calculations (cf. Tables 1 and 2). Specifically, we need the dynamic shape factor $(\chi)$, which is the ratio of the actual resistance drag of the particle to that of a sphere having the 
Table 1. (Top) Relationships of the measured quantities to the theoretical volume equivalent diameter $\left(d_{e}\right)$. An irregular particle melted and reformed as a sphere has a volume of $(\pi / 6)\left(d_{e}\right)^{3}$. (Btm) Relationships of the derived quantities $\left(L, \rho_{p}\right.$, and $\left.\rho_{L}\right)$ to the measured quantities $\left(d_{m}, d_{v a}\right.$, and $\left.\bar{m}_{L}\right)$. Terms not defined elsewhere include $F_{D}$ (the drag force), $\eta$ (the absolute viscosity of air), and $v$ (the particle velocity). (For further derivation of the relationships shown in this table, see chapter 3 of Hinds (1999) and chapters 3 and 4 of Baron and Willeke (2001)).

Relationships of the measured quantities to the theoretical volume equivalent diameter $\left(d_{e}\right)$

\begin{tabular}{|c|c|}
\hline Quantity & Equation \\
\hline \multirow[t]{2}{*}{ Dynamic shape factor, $\chi$} & $\chi=\phi($ shape,$K n(d)) \chi^{\text {shape }}=\frac{F_{D}{ }^{\text {actual }}}{F_{D}{ }^{\text {sphere }\left(d_{e}\right)}}=\frac{C_{c}\left(K n\left(d_{e}\right)\right) F_{D}{ }^{\text {actual }}}{3 \pi \eta v d_{e}}$ \\
\hline & $\begin{aligned} & \text { Examples: } \chi^{\text {shape }}=1.00 \text { for a sphere and } \chi^{\text {shape }}=1.08 \text { for a cube. } \\
& \phi(K n(d))=1 \text { for } K n<0.1 . \phi(K n(d)) \neq 1 \text { for } K n(d)>0.1 \text { (except for a sphere). } \\
& \text { We define } \chi_{a}^{\prime} \text { for } P=1 \text { atm and } 0.1<K n(d)<10, \text { for which } \chi_{a}^{\prime}=\phi(\text { shape, } K n(d)) \chi^{\text {shape }} . \\
& \text { We define } \chi_{v} \text { for } K n>10 \text {, for which } \chi_{v}=\phi(\text { shape, } K n(d)) \chi^{\text {shape }} . \\
& \text { For spherical particles, } \phi(\operatorname{shape}, K n(d)) \equiv 1 \text { and, therefore, } \chi=\chi_{a}^{\prime}=\chi_{v}=1 . \\
& \text { See further notes on the term } \phi \text { in the text. }\end{aligned}$ \\
\hline \multirow[t]{2}{*}{ Mobility diameter, $d_{m}$} & because $P=1 \mathrm{~atm}$ and $0.1<K n<10$ for submicron particles \\
\hline & inside the DMA where $\chi_{a} \equiv \chi_{a}^{\prime} \frac{C_{c}\left(K n\left(d_{m}\right)\right)}{C_{c}\left(K n\left(d_{e}\right)\right)}$ \\
\hline
\end{tabular}

Vacuum aerodynamic diameter, $d_{a}=d_{e}\left(\frac{\rho_{p}}{\rho_{0} \chi} \frac{C_{c}\left(K n\left(d_{e}\right)\right)}{C_{c}\left(K n\left(d_{v a}\right)\right)}\right)^{1 / 2} \Rightarrow d_{v a} \approx d_{e}\left(\frac{\rho_{p}}{\rho_{0} \chi_{v}} \frac{d_{v a}}{d_{e}}\right)^{1 / 2}$ at $10^{-5}$ torr inside the $\operatorname{AMS}^{*}\left(\rho_{0}=1.000 \mathrm{~g} \cdot \mathrm{cm}^{-3}\right)$ $d_{v a}$ (subscript $v$ denotes vacuum pressures, viz. $K n>10$ ) $\Rightarrow$ Rearrangement yields $d_{v a}=d_{e} \frac{\rho_{p}}{\rho_{0} \chi_{v}}$ at low pressure.

*General form: $C_{c}=1+K n(1.142+0.558 \exp (-0.999 / K n)$

At low pressure $(K n>10): C_{c} \approx 1.700 K n$

Then, $\frac{C_{c}\left(K n\left(d_{e}\right)\right)}{C_{c}\left(K n\left(d_{v a}\right)\right)} \approx \frac{d_{v a}}{d_{e}}$ at low pressure.

Mass of layer, $\bar{m}_{L}$ $\bar{m}_{L}=\frac{\pi}{6}\left(\rho_{p} d_{e}^{3}-\rho_{\text {core }} d_{\text {core }}^{3}\right)=\frac{\pi}{6} \rho_{L}\left(d_{e}^{3}-d_{\text {core }}^{3}\right)$ where $\rho_{\text {core }}=1.054 \mathrm{~g} \cdot \mathrm{cm}^{-3}$ and $d_{\text {core }}=101 \mathrm{~nm}$

Relationships of the derived quantities ( $L, \rho_{p}$, and $\left.\rho_{L}\right)$ to the measured quantities $\left(d_{m}, d_{v a}\right.$, and $\left.\bar{m}_{L}\right)$

\begin{tabular}{ll}
\hline Quantity & Equation \\
\hline Layer thickness $(L)$ & $L=\left(d_{e}-101\right) / 2=\left(d_{m} / \chi_{a}-101\right) / 2$ \\
Density of particle $\left(\rho_{p}\right)$ & $\rho_{p}=\frac{1}{d_{e}^{3}}\left(\frac{6 \bar{m}_{L}}{\pi}+\rho_{\text {core }} d_{\text {core }}^{3}\right)=\frac{\chi_{a}^{3}}{d_{m}^{3}}\left(\frac{6 \bar{m}_{L}}{\pi}+\rho_{\text {core }} d_{\text {core }}^{3}\right)=\left[\left(\frac{1}{d_{v a} \rho_{0} \chi_{v}}\right)^{3}\left(\frac{6 \bar{m}_{L}}{\pi}+\rho_{\text {core }} d_{\text {core }}^{3}\right)\right]^{-1 / 2}=\rho_{0} \chi_{a} \chi_{v} \frac{d_{v a}}{d_{m}} \quad \mathrm{~T} 1.6$ \\
Density of layer $\left(\rho_{L}\right)$ & $\rho_{L}=\frac{\bar{m}_{L}}{\frac{\pi}{6}\left(d_{e}^{3}-d_{\text {core }}^{3}\right)}=\frac{6 \bar{m}_{L} / \pi}{\left(\left(d_{m} / \chi_{a}\right)^{3}-d_{\text {core }}^{3}\right)}=\frac{6 \bar{m}_{L} / \pi}{\left(\left(\chi_{v} \rho_{0} d_{v a} / \rho_{p}\right)^{3}-d_{\text {core }}^{3}\right)} \quad \mathrm{T} 1.7$ \\
\hline
\end{tabular}


Table 2. Relationships among $d_{m}, d_{v a}$, and $\bar{m}_{L}$.

\begin{tabular}{|c|c|c|c|}
\hline & $d_{v a}$ & $d_{m}$ & $\overline{\boldsymbol{m}}_{L}$ \\
\hline$d_{v a}$ & - & $d_{m}=\frac{\rho_{0} \chi_{a} \chi_{v}}{\rho_{p}} d_{v a}$ & $\bar{m}_{L}=\frac{\pi}{6}\left(\frac{\left(\rho_{0} \chi_{v} d_{v a}\right)^{3}}{\rho_{p}^{2}}-\rho_{\text {core }} d_{\text {core }}^{3}\right.$ \\
\hline$d_{m}$ & $d_{v a}=\frac{\rho_{p}}{\rho_{0} \chi_{a} \chi_{v}} d_{m}$ & 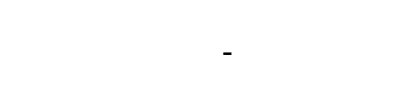 & $\bar{m}_{L}=\frac{\pi}{6}\left(\frac{\rho_{p} d_{m}^{3}}{\chi_{a}^{3}}-\rho_{\text {core }} d_{\text {core }}^{3}\right)$ \\
\hline$\overline{\boldsymbol{m}}_{L}$ & $d_{v a}=\frac{1}{\rho_{0} \chi_{v}}\left[\rho_{p}^{2}\left(\frac{6 \bar{m}_{L}}{\pi}+\rho_{\text {core }} d_{\text {core }}^{3}\right)\right.$ & $d_{m}=\chi_{a}\left[\frac{1}{\rho_{p}}\left(\frac{6 \bar{m}_{L}}{\pi}+\rho_{\text {core }} d_{\text {core }}^{3}\right.\right.$ & - \\
\hline
\end{tabular}

Table 3. Summary of the results of six experiments. Given are (1) the primary measurements of dynamic shape factor $(\chi)$, mobility diameter $\left(d_{m}\right)$, vacuum aerodynamic diameter $\left(d_{v a}\right)$, aerosol layer mass $\left(m_{L}\right)$, and particle number concentration measured by the SMPS $(N)$ and (2) the derived quantities of layer thickness $(L)$, particle layer mass $\left(\bar{m}_{L}\right)$, particle density $\left(\rho_{p}\right)$, and layer density $\left(\rho_{L}\right)$. The uncertainties (one sigma) shown for the derived quantities are based upon the uncertainties of the primary measurements (see Sect. 3.3). (Top) Results are shown for unreacted particles. (Btm) Results are shown for the same particles having 1.0 normalized ozone exposure, which is defined as an ozone exposure $\left(\mathrm{P}_{\mathrm{O} 3} t\right)$ such that $m_{\mathrm{OL}} /\left(m_{\mathrm{OL}}\right)_{0}=0.05$ where $m_{\mathrm{OL}}$ is the mass of oleic acid in the coating, $\mathrm{P}_{\mathrm{O} 3}$ is the partial pressure of ozone, and $t$ is the reaction time. ${ }^{*}$ Note added in proof: The covariance of $m_{L}$ and $N$ (see Sect. A7) suggest that this number refers to $2 \%$ precision instead of $2 \%$ accuracy. Figure $6 \mathrm{~b}$ shows this precision. The high precision is obtained because of the similarity between the test system (oleic acid and its ozonolysis products) and the calibration system (oleic acid). The absolute accuracy of $m_{L}$ cannot be better than the combined accuracies of $N(5 \%)$ and $d_{m}(1 \%)$ because of the calibration procedure (Eq. A2.1). In the experiments reported in this paper, which are focused on density, the accuracy of the calibration cancels out, as shown by the sensitivity study in Table 6 .

\begin{tabular}{|c|c|c|c|c|c|c|c|c|c|c|c|c|}
\hline \multirow[b]{2}{*}{ Experiment } & \multicolumn{5}{|c|}{ Measurements } & \multicolumn{7}{|c|}{ Derived Quantities } \\
\hline & $\chi$ & $\begin{array}{c}\boldsymbol{d}_{\boldsymbol{m}} \\
(\mathrm{nm})\end{array}$ & $\begin{array}{c}\boldsymbol{d}_{\boldsymbol{v a}} \\
(\mathrm{nm})\end{array}$ & $\begin{array}{c}\boldsymbol{m}_{\boldsymbol{L}} \\
\left(\mu \mathrm{g} \cdot \mathrm{m}^{-3}\right)\end{array}$ & $\begin{array}{c}N \\
\left(\# \mathrm{~cm}^{-3}\right)\end{array}$ & $\begin{array}{c}\boldsymbol{L} \\
(\mathrm{nm})\end{array}$ & $\begin{array}{c}\overline{\boldsymbol{m}}_{\boldsymbol{L}} \\
\left(10^{-15} \mathrm{~g}\right)\end{array}$ & $\begin{array}{c}\overline{\boldsymbol{m}}_{\boldsymbol{L}} \\
\left(10^{-15} \mathrm{~g}\right)\end{array}$ & $\begin{array}{c}\rho_{p} \\
\left(\mathrm{~g} \cdot \mathrm{cm}^{-3}\right)\end{array}$ & $\begin{array}{c}\rho_{p} \\
\left(\mathrm{~g} \cdot \mathrm{cm}^{-3}\right)\end{array}$ & $\begin{array}{c}\rho_{L} \\
\left(\mathrm{~g} \cdot \mathrm{cm}^{-3}\right)\end{array}$ & $\begin{array}{c}\rho_{L} \\
\left(\mathrm{~g} \cdot \mathrm{cm}^{-3}\right)\end{array}$ \\
\hline$\# 1$ & 1.00 & 101 & 107 & 0.0 & 9000 & 0 & - & - & $1.059 \pm 0.015$ & $1.055 \pm 0.032$ & - & - \\
\hline$\# 2$ & 1.00 & 117 & 118 & 2.5 & 9100 & $8.0 \pm 0.6$ & $0.28 \pm 0.01$ & $0.28 \pm 0.02$ & $1.009 \pm 0.014$ & $1.007 \pm 0.035$ & $0.928 \pm 0.036$ & $0.926 \pm 0.094$ \\
\hline$\# 3$ & 1.00 & 123 & 122 & 3.7 & 9350 & $11.0 \pm 0.6$ & $0.40 \pm 0.02$ & $0.40 \pm 0.02$ & $0.992 \pm 0.014$ & $0.992 \pm 0.037$ & $0.916 \pm 0.028$ & $0.918 \pm 0.080$ \\
\hline$\# 4$ & 1.00 & 137 & 133 & 6.9 & 9400 & $18.0 \pm 0.7$ & $0.74 \pm 0.04$ & $0.74 \pm 0.03$ & $0.971 \pm 0.014$ & $0.970 \pm 0.041$ & $0.916 \pm 0.021$ & $0.915 \pm 0.067$ \\
\hline$\# 5$ & 1.00 & 151 & 144 & 10.8 & 9500 & $25.0 \pm 0.8$ & $1.14 \pm 0.06$ & $1.15 \pm 0.04$ & $0.954 \pm 0.013$ & $0.949 \pm 0.045$ & $0.911 \pm 0.018$ & $0.904 \pm 0.063$ \\
\hline \#6 & 1.00 & 161 & 152 & 14.1 & 9500 & $30.0 \pm 0.8$ & $1.49 \pm 0.08$ & $1.49 \pm 0.05$ & $0.944 \pm 0.013$ & $0.942 \pm 0.047$ & $0.908 \pm 0.017$ & $0.906 \pm 0.061$ \\
\hline Accuracy & $0 \%$ & $1 \%$ & $1 \%$ & $2 \%^{*}$ & $5 \%$ & & & & & & & \\
\hline Comment & $A M S$ & $D M A$ & $A M S$ & $A M S$ & $S M P S$ & eq T1.5 & $e q 1 a$ & $e q 1 b$ & $e q 2 a$ & $e q 2 b$ & eq $3 a$ & $e q 3 b$ \\
\hline
\end{tabular}

After 1.0 normalized ozone exposure

\begin{tabular}{|c|c|c|c|c|c|c|c|c|c|c|c|c|}
\hline \multirow[b]{2}{*}{ Experiment } & \multicolumn{5}{|c|}{ Measurements } & \multicolumn{7}{|c|}{ Derived Quantities } \\
\hline & $\chi$ & $\begin{array}{c}\boldsymbol{d}_{\boldsymbol{m}} \\
(\mathrm{nm})\end{array}$ & $\begin{array}{c}\boldsymbol{d}_{\boldsymbol{v a}} \\
(\mathrm{nm})\end{array}$ & $\begin{array}{c}\boldsymbol{m}_{\boldsymbol{L}} \\
\left(\mu \mathrm{g} \cdot \mathrm{m}^{-3}\right)\end{array}$ & $\begin{array}{c}N \\
\left(\# \mathrm{~cm}^{-3}\right)\end{array}$ & $\begin{array}{c}\boldsymbol{L} \\
(\mathrm{nm})\end{array}$ & $\begin{array}{c}\overline{\boldsymbol{m}}_{\boldsymbol{L}} \\
\left(10^{-15} \mathrm{~g}\right)\end{array}$ & $\begin{array}{c}\overline{\boldsymbol{m}}_{\boldsymbol{L}} \\
\left(10^{-15} \mathrm{~g}\right)\end{array}$ & $\begin{array}{c}\rho_{p} \\
\left(\mathrm{~g} \cdot \mathrm{cm}^{-3}\right)\end{array}$ & $\begin{array}{c}\rho_{p} \\
\left(\mathrm{~g} \cdot \mathrm{cm}^{-3}\right)\end{array}$ & $\begin{array}{c}\rho_{L} \\
\left(\mathrm{~g} \cdot \mathrm{cm}^{-3}\right)\end{array}$ & $\begin{array}{c}\rho_{L} \\
\left(\mathrm{~g} \cdot \mathrm{cm}^{-3}\right)\end{array}$ \\
\hline$\# 1$ & 1.00 & 101 & 107 & 0.0 & 9000 & 0 & - & - & $1.059 \pm 0.015$ & $1.055 \pm 0.031$ & - & - \\
\hline$\# 2$ & 1.00 & 115 & 119 & 1.9 & 7500 & $7.0 \pm 0.6$ & $0.25 \pm 0.01$ & $0.25 \pm 0.02$ & $1.053 \pm 0.015$ & $1.053 \pm 0.036$ & $1.052 \pm 0.050$ & $1.056 \pm 0.121$ \\
\hline$\# 3$ & 1.00 & 118 & 124 & 2.7 & 8100 & $9.0 \pm 0.6$ & $0.34 \pm 0.02$ & $0.34 \pm 0.02$ & $1.059 \pm 0.015$ & $1.055 \pm 0.038$ & $1.069 \pm 0.041$ & $1.060 \pm 0.103$ \\
\hline$\# 4$ & 1.00 & 131 & 135 & 5.2 & 8200 & $15.0 \pm 0.7$ & $0.67 \pm 0.04$ & $0.67 \pm 0.03$ & $1.053 \pm 0.015$ & $1.051 \pm 0.044$ & $1.053 \pm 0.028$ & $1.050 \pm 0.081$ \\
\hline$\# 5$ & 1.00 & 142 & 150 & 8.1 & 8000 & $21.0 \pm 0.7$ & $1.04 \pm 0.06$ & $1.05 \pm 0.04$ & $1.077 \pm 0.015$ & $1.074 \pm 0.050$ & $1.091 \pm 0.024$ & $1.086 \pm 0.079$ \\
\hline$\# 6$ & 1.00 & 148 & 158 & 9.8 & 7900 & $24.0 \pm 0.8$ & $1.26 \pm 0.07$ & $1.27 \pm 0.04$ & $1.081 \pm 0.015$ & $1.078 \pm 0.052$ & $1.094 \pm 0.023$ & $1.089 \pm 0.077$ \\
\hline Accuracy & $0 \%$ & $1 \%$ & $1 \%$ & $2 \%^{*}$ & $5 \%$ & & & & & & & \\
\hline Comment & $A M S$ & $D M A$ & $A M S$ & $A M S$ & SMPS & eq T1.5 & eq $1 a$ & $e q 1 b$ & $e q 2 a$ & $e q 2 b$ & eq $3 a$ & $e q 3 b$ \\
\hline
\end{tabular}

same volume and velocity (Eq. T1.1). Unfortunately, there is no unique relationship between lift and drag forces. For example, although neither a cube nor a sphere has a lift force (Liu et al., 1995a), the drag force of a cube is $8 \%$ greater than that of a sphere (Hinds, 1999). Nevertheless, given our experimental setup employing PSL cores, a small beam divergence is sufficient to conclude that we have spherical particles. 
The dynamic shape factor differs whether the Knudsen number ${ }^{2}(K n)$ is greater than 10 (e.g., vacuum conditions and $100-\mathrm{nm}$ particles) or $0.1<K n<10$ (e.g., inside the DMA, Jimenez et al., 2003a). We can separate the effects of shape from pressure by writing $\chi=\phi($ shape, $K n(d)) \chi^{\text {shape }}$. Except for certain streamlined shapes, $\chi^{\text {shape }}>1.0$. The term $\phi$ arises from the shape dependence of the Cunningham slip correction factor, as follows: $C_{c}\left(\right.$ shape, $\left.K n\left(d_{e}\right)\right)=\phi($ shape, $K n(d)) C_{c}\left(K n\left(d_{e}\right)\right)$ where, for nonspherical particles, a useful concept is the volume equivalent diameter $\left(d_{e}\right)$, which corresponds to the volume of a nonspherical particle reformed into a spherical particle. To indicate that $P=1$ atm and $0.1<K n<10$, which are the conditions inside the DMA for submicron particles, we employ the designation $\chi_{a}^{\prime}$ (i.e., $\chi_{a}^{\prime}=\phi \chi^{\text {shape }}=\chi^{\text {shape }}$ ), which we call the atmospheric dynamic shape factor. For $K n>10$, the correction $\phi$ is not negligible. To indicate that $K n>10$, we employ the designation $\chi_{v}$ (i.e., $\left.\chi_{v}=\phi \chi^{\text {shape }}\right)$. By definition, $\phi($ sphere $)=1$ and $\chi^{\text {shape }}($ sphere $)=1$. Therefore, $\chi=\chi_{a}^{\prime}=\chi_{v}=1$ for spherical particles.

\subsection{Measurement of electric mobility diameter $\left(d_{m}\right)$}

The electric mobility diameter of a particle of arbitrary shape equals the diameter of a sphere having the same electric mobility. For example, a particle of arbitrary shape and charge that has a mobility diameter of $100 \mathrm{~nm}$ behaves electrophoretically as a 100-nm spherical particle having one charge. Importantly, mobility diameter is independent of particle density.

The electric mobility diameters of the test aerosol particles are measured via a TSI model 3071 differential mobility analyzer (software version 3.2), which incorporates an aerosol neutralizer (krypton-85 source). This instrument operates by the principle of electrophoresis to classify positively charged particles. A 10:1 sheath-to-polydisperse aerosol flow is used. A charge correction algorithm assuming a Boltzmann distribution is employed, although the percentage of multiply charged particles is not significant for the particle diameters of 100 to $150 \mathrm{~nm}$ employed in the experiments. For this size range, an impactor is also unnecessary.

\subsection{Measurement of vacuum aerodynamic diameter $\left(d_{v a}\right)$}

The aerodynamic diameter of a particle of arbitrary shape and density is the diameter of a spherical particle of unit density $\left(\rho_{0}=1.000 \mathrm{~g} \cdot \mathrm{cm}^{-3}\right)$ having an identical settling velocity as the test particle. For example, a particle having an aerodynamic diameter of $100 \mathrm{~nm}$ has a settling velocity equal to that of a non-evaporating, 100-nm spherical particle of unit density, regardless of the particle's true shape, density, or phys-

\footnotetext{
${ }^{2}$ The Knudsen number $K n$ given by $K n=2 \lambda / d \approx 13.4 /(d[\mu m] P[k P a])$ defines the continuum $(K n<0.1)$, transition $(0.1<K n<10)$, and free molecular $(K n>10)$ regimes
}

ical size. Because settling velocity depends on pressure via the Cunningham slip correction factor $\left(C_{c}\right)$ (Table 1), the description of an aerodynamic diameter is incomplete without also considering $K n$ (cf. Sect. 2.1). The aerodynamic diameter measured in the AMS is under conditions of $K n>10$, and we use the term $d_{v a}$ for these conditions (Jimenez et al., 2003a). As a result of $K n>10$, the relationships shown for $d_{v a}$ in Tables 1 and 2 differ from equations used for $d_{a}$ measured when $K n<10$ (Murphy et al., 2004).

Although the aerodynamic diameter is strictly defined in reference to a settling velocity, conveniently the velocity of a particle accelerated through a critical-flow pressure drop has an inverse power dependence on aerodynamic diameter, provided that the particle Reynolds number is below unity (Baron and Willeke, 2001). The Reynolds number is below unity for submicron particles at the inlet pressure of the critical orifice of the AMS (Jayne et al., 2000).

A time-of-flight (TOF) measurement inside the AMS is employed to determine particle velocity and, therefore, the vacuum aerodynamic diameter. Specifically, after entering the AMS through a $100-\mu \mathrm{m}$ critical orifice, the particles are accelerated and focused into a narrow beam (ca. $1 \mathrm{~mm}$ ) by passing through an aerodynamic lens (Jayne et al., 2000). A spinning chopper wheel $(180 \mathrm{~Hz}$ and $0.50 \%$ duty cycle) placed at the exit of the aerodynamic lens forms pulses of particles and defines time zero within $28 \mu$ s uncertainty. The particle beam impacts onto a vaporizer, which is a resistively heated, 3.8-mm hotplate $\left(\mathrm{ca} .350^{\circ} \mathrm{C}\right)$. The semi-volatile constituents of the particle are flash vaporized upon striking the hot surface, the vapors are ionized by electron impact, and the ions are detected by quadrupole mass spectrometry (MS). The time difference between detection at the MS and time zero yields the particle time of flight, from which the velocity of the particle is calculated and the vacuum aerodynamic diameter is obtained. For example, 100-nm particles have a time of flight of approximately $5 \mathrm{~ms}$. The vaporizationionization-detection process usually occurs much faster than the particle flight time, although in some cases particle vaporization can be slow enough to measurably increase the apparent flight time and thus lead to an overestimate of the vacuum aerodynamic diameter. A tuned value of $41 \mathrm{amu}$ is used for the time-of-flight studies of oleic acid and its ozonolysis products.

\subsection{Measurement of aerosol layer mass $\left(m_{L}\right)$}

The operation principles to obtain quantitative aerosol mass loadings $\left(\mu \mathrm{g} \cdot \mathrm{m}^{-3}\right)$ using the AMS and given a stable test aerosol are described in detail by Jayne et al. (2000), Jimenez et al. (2003b), and Katrib et al. (2004). In brief, the quadrupole mass spectrometer is tuned from 10 to $300 \mathrm{amu}$ to provide a mass spectrum of the volatilized constituents of the particle ensemble. The total particle mass loading is obtained based upon the calibrated response of the MS signal intensity to mass. The measured aerosol mass loadings arise from the 
mass present in the semi-volatile coatings surrounding the PSL cores: the PSL core particles do not volatilize under the usual operating conditions of $350^{\circ} \mathrm{C}$ for the hotplate, and the gas-phase species are removed by the pumping employed to maintain vacuum conditions.

\subsection{Measurement of particle number density $(N)$}

The number concentration of the particles is determined by SMPS measurements. Specifically, $\mathrm{d} N / \mathrm{d} \log d_{m}$ is integrated across the mode at or just above $100 \mathrm{~nm}$ (depending on layer thickness). A nanoparticle mode from 50 to $90 \mathrm{~nm}$, which may result from homogeneous nucleation of the oleic acid vapor during the coating process or from deposition of the oleic acid vapor onto sub-10 nm impurities in the atomized water, lies below the lower limit of the integration. (We also tested the approach of measuring $N$ via the single-particle capability of the AMS. We found, however, that this approach is less accurate in our experimental setup because the small layer mass of ca. $10^{-15} \mathrm{~g}$ on individual particles implies that a fraction of the individual particles fails to trigger a counting threshold on the AMS. Integrated properties such as aerosol layer mass are, however, still accurately measured.)

\subsection{Calculation of layer thickness $(L)$}

Under the assumption of a uniform coating on spherical particles, the increase of particle geometric diameter beyond that of the PSL core is twice the layer thickness of the organic coating. Equation (T1.5) shows that $L=\left(d_{m} / \chi_{a}-101\right) / 2$.

\subsection{Calculation of particle layer mass $\left(\bar{m}_{L}\right)$}

We calculate the average layer mass per particle $\left(\bar{m}_{L}\right)$ by two independent methods. In the first method, measurements of aerosol layer mass and particle number concentration (Sects. 2.4 and 2.5) are combined to yield:

$\bar{m}_{L}\left(m_{L}, N\right)=m_{L} / N$

In the second method, measurements of vacuum aerodynamic diameter, mobility diameter, and dynamic shape factor are combined using Eqs. (T1.2), (T1.3), and (T1.4) to yield the following equation:

$\bar{m}_{L}\left(d_{v a}, d_{m}, \chi_{a}, \chi_{v}\right)=\frac{\pi}{6}\left(\rho_{0} d_{v a} d_{m}^{2} \chi_{v} / \chi_{a}^{2}-\rho_{\text {core }} d_{c o r e}^{3}\right)(1 \mathrm{~b})$

\subsection{Calculation of particle $\left(\rho_{p}\right)$ and layer $\left(\rho_{L}\right)$ densities}

The measurements can be employed to calculate the density of the particle and of the organic outer layer. There are two independent methods for doing so. Particle density can be calculated by $\rho_{p}=f\left(d_{v a}, d_{m}, \chi_{a}, \chi_{v}\right)$ (Eq. 2a) or $\rho_{p}=f\left(\bar{m}_{L}, d_{m}, \chi_{a}\right)$ (Eq. 2 b)(cf. Eq. T1.6), as follows:

$\rho_{p}\left(d_{v a}, d_{m}, \chi_{a}, \chi_{v}\right)=\rho_{0} \chi_{a} \chi_{v} \frac{d_{v a}}{d_{m}}$
$\rho_{p}\left(\bar{m}_{L}, d_{m}, \chi_{a}\right)=\frac{\chi_{a}^{3}}{d_{m}^{3}}\left(\frac{6 \bar{m}_{L}}{\pi}+\rho_{\text {core }} d_{\text {core }}^{3}\right)$

The quantitative results of these two independent methods can be compared to each other. In Eq. (2b), we use Eq. (1a) to evaluate $\bar{m}_{L}$.

Particle layer density is determined by two independent methods through the use of Eqs. (T1.2), (T1.4), and (2a), as follows:

$\rho_{L}\left(d_{v a}, d_{m}, \chi_{a}, \chi_{v}\right)=\frac{\left(\rho_{0} \chi_{a} \chi_{v} d_{v a} / d_{m}-\rho_{\text {core }} \chi_{a}^{3}\left(d_{\text {core }} / d_{m}\right)^{3}\right)}{\left(1-\chi_{a}^{3}\left(d_{\text {core }} / d_{m}\right)^{3}\right)}$

$\rho_{L}\left(\bar{m}_{L}, d_{m}, \chi_{a}\right)=\frac{6 \bar{m}_{L} / \pi}{\left(\left(d_{m} / \chi_{a}\right)^{3}-d_{\text {core }}^{3}\right)}$

An effective density, which relates $d_{m}$ to $d_{a}$, is commonly reported in the literature (DeCarlo et al., 2004). The effective density evaluates as $\rho_{e}=\rho_{p} / \chi_{a}^{3}$ in the governing equation $d_{a}^{2} \rho_{0}=\rho_{e} d_{m}^{2}$ when $d_{a}$ and $d_{m}$ are measured for $0.1<K n<10$ (e.g., when aerodynamic diameter is determined by impaction at $1 \mathrm{~atm}$ ) (Kelly and McMurry, 1992). Under our experimental conditions employing the vacuum aerodynamic diameter, however, the effective density evaluates as $\rho_{v e}=\rho_{p} / \chi_{a} \chi_{v}$ in the governing equation $d_{v a} \rho_{0}=\rho_{v e} d_{m}$ for the measured $d_{v a}$ and $d_{m}$ (cf. equations in Table 2). There is, therefore, a change in the governing equation from a quadratic to a linear form depending on experimental conditions. In the analysis of this paper, we do not employ an effective density because we determine that we have spherical, nonporous particles $(\chi=1)$, in which case the effective density equals the density.

\section{Results and discussion}

\subsection{Spherical particle shape}

The divergence of the particle beam inside the AMS, which is defined as $90 \%$ of the integrated transmission in agreement with Liu et al. (1995a), is an indicator of particle shape. For example, the beam profiles of several calibration particles show that spherical particles, such as liquid oleic acid or aqueous sodium chloride, have the narrowest Gaussian profiles (Fig. 2a). In comparison, particle beams of unreacted and reacted coated particles have similar Gaussian profiles, regardless of layer thickness. We therefore conclude that these particles are also spherical. The volume fraction of the inert PSL core is high, which is important for maintaining sphericity. Consistent with this finding, we assume in our analysis that the particles are radially symmetric and nonporous.

The solid angle of beam divergence for spherical particles is approximately $0.40 \times 10^{-5} \mathrm{sr}$ in our apparatus, which can be compared to $1.69 \times 10^{-5} \mathrm{sr}$ for spherical $100-\mathrm{nm}$ dioctyl sebacate (DOS) particles (density of $0.912 \mathrm{~g} \mathrm{~cm}^{-3}$ ) in the 
aerodynamic lens of Liu et al. (1995b). Although the beam divergences are similar, the small differences may arise from differences in the design of the aerodynamic lens installed in the AMS compared to the one employed by Liu et al. (1995b). In contrast to the spherical particles, the beam divergence in our apparatus is approximately $1.6 \times 10^{-5} \mathrm{sr}$ for crystalline sodium chloride particles. Liu et al. (1995b) estimate that $\Omega=10.6 \times 10^{-5} \mathrm{sr}$ for $100-\mathrm{nm}$ crystalline sodium chloride particles. Given this evidence of nonvanishing lift force, Liu et al. (1995b) infer that the crystalline particles are imperfect cubes. Liu et al. generate crystalline particles via evaporation of aqueous particles having a primary diameter of 10-15 $\mu \mathrm{m}$ (Collision atomizer) as compared to the submicron primary particles (TSI 3076) of this study. The different primary sizes may affect the morphology of the dried particles. Liu et al. (1995b) also discuss an exact transformation from a lateral beam profile to a gaussian beam divergence. We did not carry out this detailed analysis, so the stated beam divergences are approximate.

We can test our supposition that the calibration liquid particles are spherical. Specifically, the dynamic shape factor can be calculated using Eqs. (T1.1-T1.3) as:

$$
\begin{aligned}
& \chi^{\text {shape }}= \\
& {\left[\frac{\rho_{p} d_{m} / \rho_{0} d_{v a}}{\phi(\text { shape }, K n>10) \phi(\text { shape }, 0.1<K n<10)} \frac{C_{c}\left(K n\left(d_{e}\right)\right)}{C_{c}\left(K n\left(d_{m}\right)\right)}\right]^{1 / 2}}
\end{aligned}
$$

In the case of pure oleic acid particles (i.e., devoid of a PSL core), we know $\rho_{p}=0.895 \mathrm{~g} \cdot \mathrm{cm}^{-3}$. When we measure a mobility diameter of $350 \mathrm{~nm}$, we correspondingly measure a vacuum aerodynamic diameter of $315 \mathrm{~nm}$. Therefore, given $\phi($ shape, $K n)=1$ and $d_{m}=d_{e}$ (both true for spheres), we calculate that $\chi^{\text {shape }}=1.00$.

Figure $2 \mathrm{~b}$ shows that neat PSL particles diverge slightly, implicating a slightly nonspherical shape, which could arise because of impurities that adsorb on the PSL when atomizing an aqueous suspension of the PSL particles. This observation is important because the time of flight of these particles is employed to calibrate the aerodynamic diameter of the AMS, for which $\chi=1$ is assumed. Similarly, the SMPS flows are adjusted for maximum transmission of these PSL particles when the voltage is tuned to correspond to a 101-nm mobility diameter. The reliability of this approach assumes that the mobility diameter corresponds to the geometric diameter of a sphere. The effects on our results of these uncertainties in the AMS and SMPS calibrations are discussed in the appendix. A 2-nm coating of oleic acid on the PSL particles is sufficient to restore a spherical shape (Fig. 2b).

3.2 Increase in particle layer density as a result of chemical reaction

Two distinct and independent methods are available to us to calculate particle density ( $\rho_{p}$; Eqs. $2 \mathrm{a}$ and $2 \mathrm{~b}$ ) and layer density ( $\rho_{L}$; Eqs. $3 \mathrm{a}$ and $\left.3 \mathrm{~b}\right)$. The first method, given by

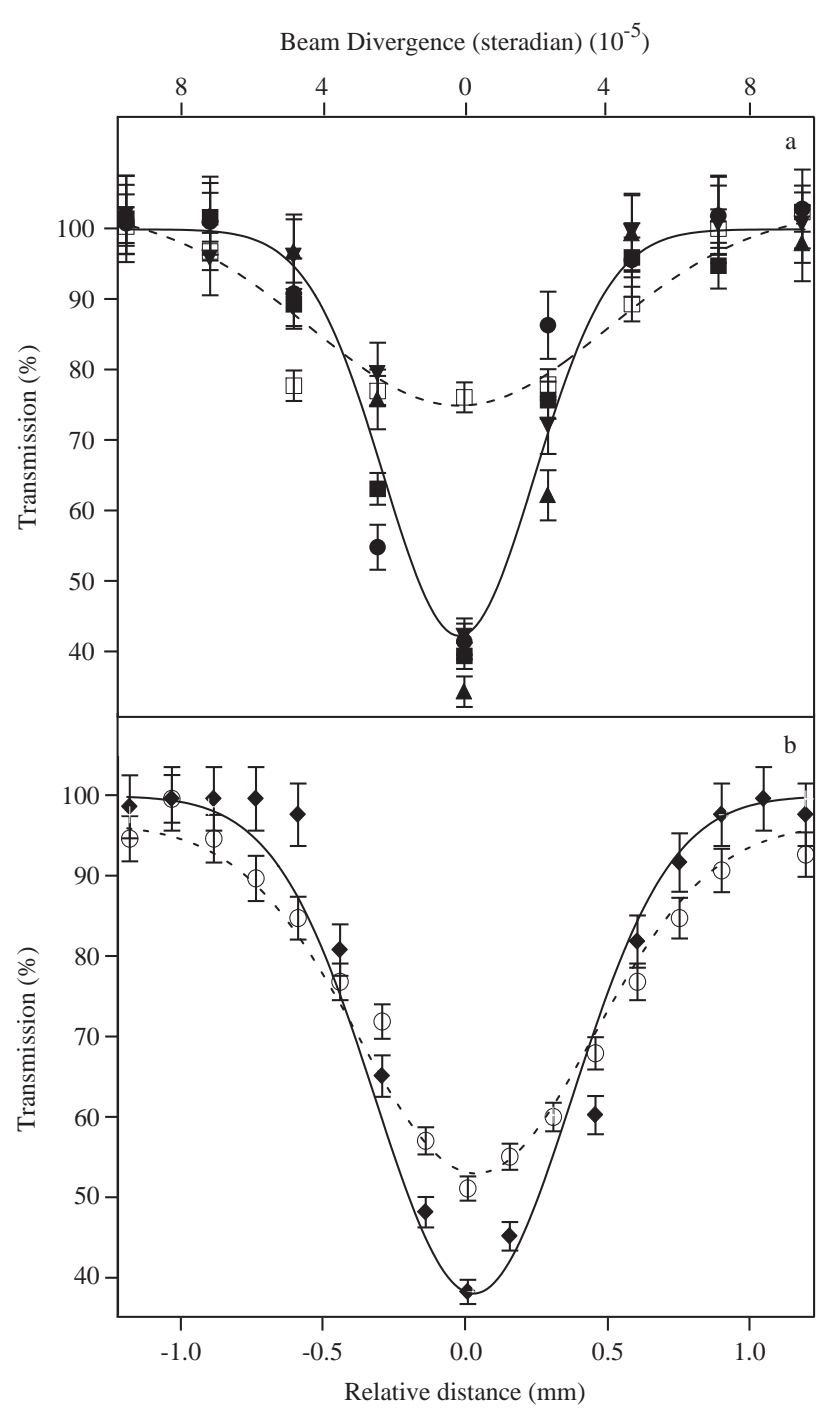

Fig. 2. Beam profiles. Transmission is the relative signal intensity at the electron multiplier when $0.3 \mathrm{~mm}$ of the particle beam is blocked. The upper axis indicates the corresponding solid angle of beam divergence. (a) Unreacted ( $\boldsymbol{\Delta}$ ) and reacted (viz. 1.0 normalized ozone exposure) ( $\boldsymbol{\nabla})$ particles having oleic acid coatings and polystyrene latex cores. Also shown are the beam profiles for pure oleic acid $(\bullet)$, aqueous sodium chloride ( $80 \%$ RH) (ם), and crystalline sodium chloride (30\% RH) $(\square)$ aerosol particles. Conditions: $d_{v a}=130 \mathrm{~nm}$. (b) PSL particles having no coating (o) $\left(d_{v a}=107 \mathrm{~nm}\right)$ compared to those having a thin oleic acid coating $(\diamond)\left(d_{v a}=111 \mathrm{~nm}\right)$. (We use an AMS vaporizer temperature of $350^{\circ} \mathrm{C}$ for the study of oleic acid, $850^{\circ} \mathrm{C}$ for the study of sodium chloride, and $900^{\circ} \mathrm{C}$ for the study of polystyrene latex.)

Eqs. (2a) and (3a), is based upon measurements of mobility and aerodynamic diameters. Figure 3 provides an example of measurements of mobility and aerodynamic diameters and their changes upon ozone exposure. The diameters $d_{m}$ and $d_{v a}$ are initially 151 and $154 \mathrm{~nm}$, respectively. Based upon Eq. (T1.5), the oleic acid layer thickness is $25 \mathrm{~nm}$. The 

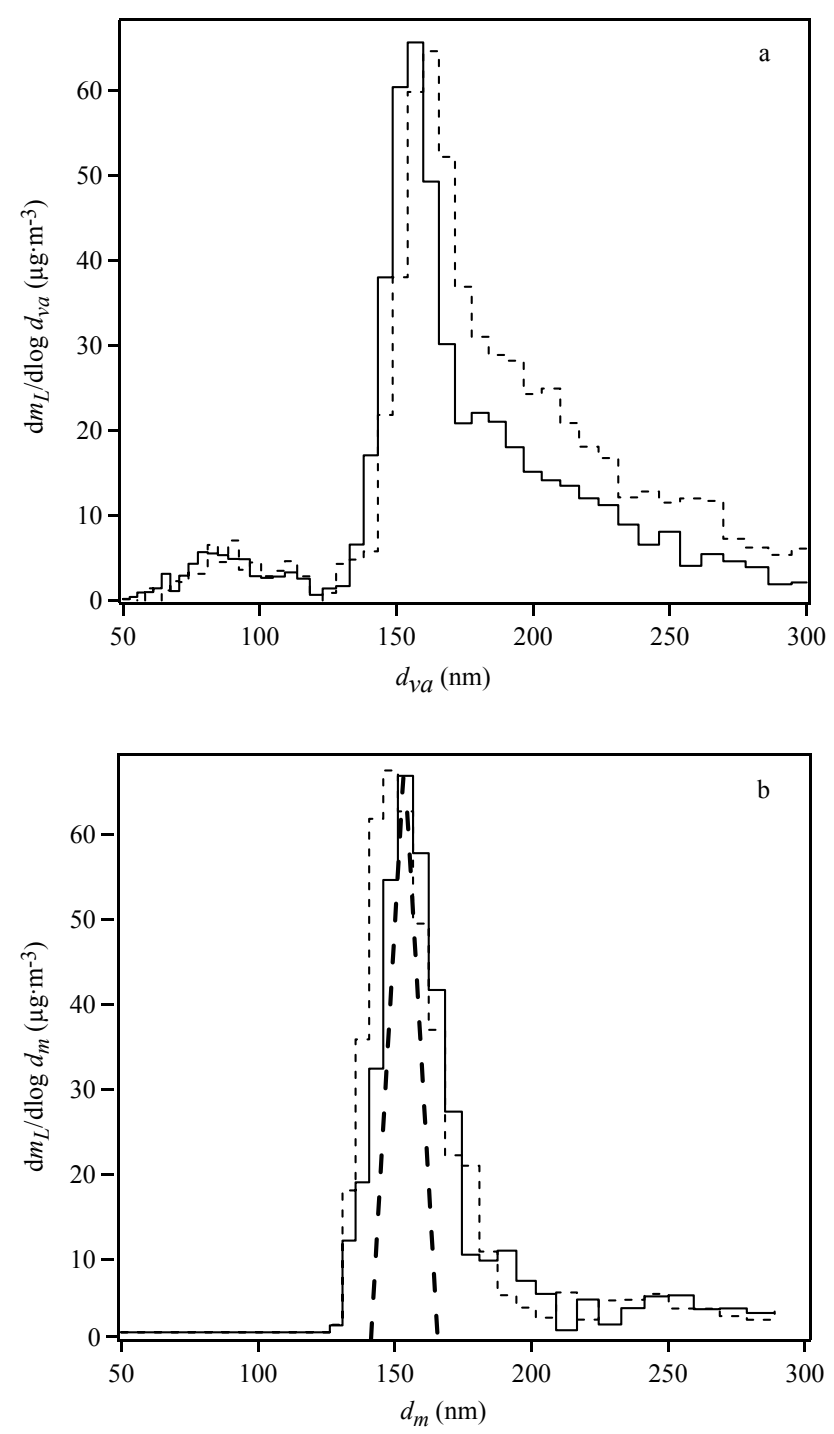

Fig. 3. (a) Aerodynamic diameter and (b) mobility diameter mass size distributions of unreacted particles (solid line) and particles after 0.6 normalized ozone exposure (dashed line). The theoretical DMA transfer function for our flow conditions is shown as a heavy dashed line. The $\mathrm{d} N / \mathrm{d} \log d_{m}$ measurements of the SMPS are transformed into the plot of $\mathrm{d} m_{L} / \mathrm{d} \log d_{m}$ shown in $b$ by using $m_{L}=0$ for $d_{m}<d_{\text {core }}$ and Eqs. (1a) and (3b) for $d_{m} \geq d_{\text {core }}$. We use $\rho_{L}=0.895$ in Eq. (3b) for unreacted particles and $\rho_{L}=1.05$ for reacted particles, as determined by application of Eq. (3a). The good agreement on the scale of the y-axes between (a) and (b) is noteworthy. Conditions: initial 25-nm oleic acid coating on polystyrene latex cores; AMS tuned to $41 \mathrm{amu}$ for $d_{v a}$ measurements.

diameters $d_{m}$ and $d_{v a}$ change to 141 and 160 , respectively, upon 0.6 normalized ozone exposure. (95\% loss of oleic acid loss is defined as 1.0 normalized ozone exposure.) The results for various layer thickness at 0.0 and 1.0 normalized ozone exposure are reported in Table 3. The layer thickness of the reacted particles decrease by ca. $25 \%$ at 1.0 normalized ozone exposure, which is consistent with a concomi-

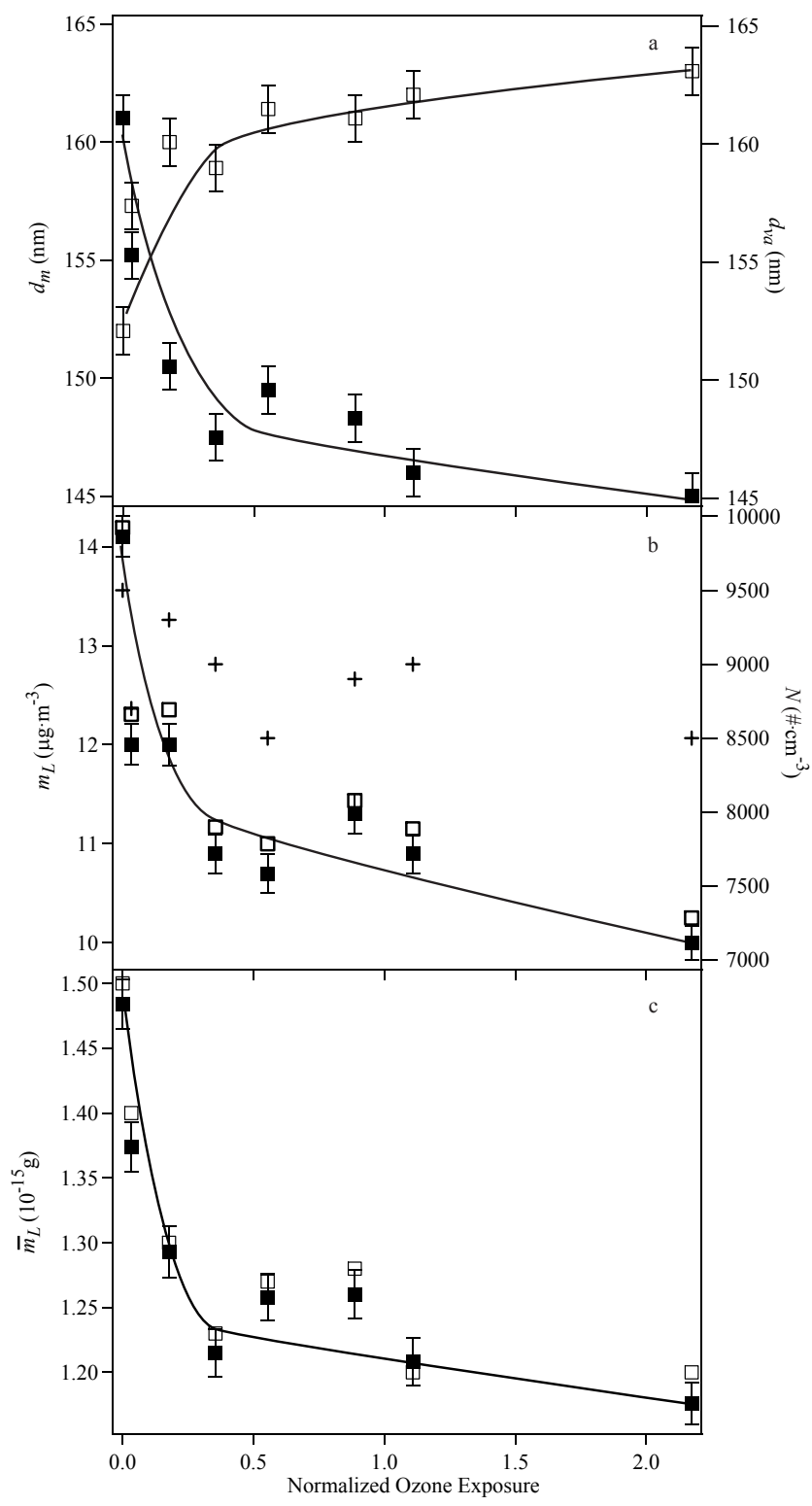

Fig. 4. (a) Variation of the aerodynamic and the mobility diameters with increasing normalized ozone exposure. Key: $d_{m}(\boldsymbol{\square})$ and $d_{v a}(\square)$. (b) Measured/predicted aerosol layer mass with increasing normalized ozone exposure. Also shown is the measured particle number density. Key: $m_{L}$ measured $(\boldsymbol{\square}), m_{L}$ predicted by combining Eqs. (1a) and (1b) ( $\square$ ), and measured $N(+)$. (c) Calculated and predicted particle layer mass with increasing normalized ozone exposure. Key: $\bar{m}_{L}$ calculated by using Eq. (1a) (口) and $\bar{m}_{L}$ predicted by Eq. (1b) ( $\square$ ). Conditions (a), (b), and (c): initial 30-nm oleic acid coating on polystyrene latex cores.

tant decrease in aerosol layer mass $\left(m_{L}\right)$ due to the evaporation of volatile reaction products such as 1-nonanal (Moise and Rudich, 2002; Thornberry and Abbatt, 2004; Hearn and Smith, 2004). The changes in $d_{m}$ and $d_{v a}$ with increasing ozone exposure are shown in Fig. 4a for an initially 30-nm layer thickness. The general finding is that, regardless of 

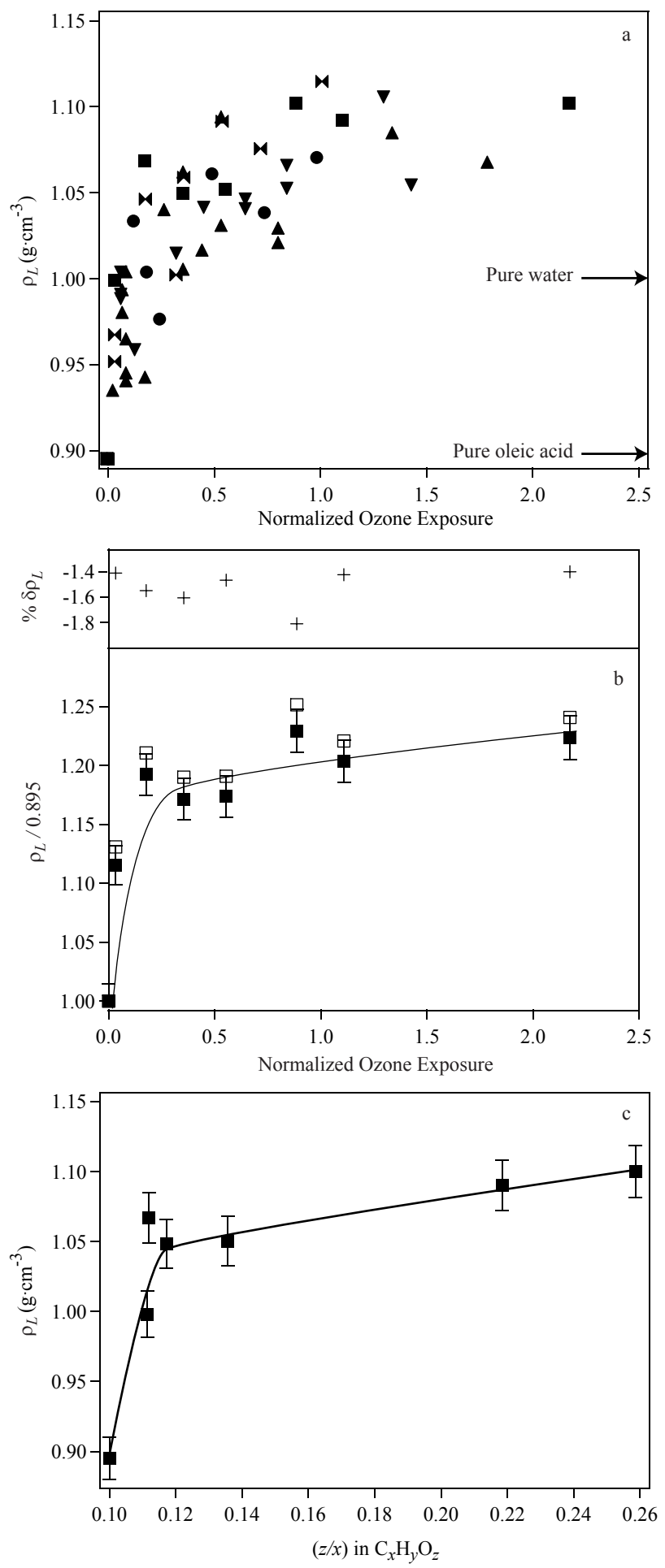

Fig. 5. (a) Layer density for increasing normalized ozone exposure for 8 $\mathrm{nm}(\bullet), 11-\mathrm{nm}(\boldsymbol{\Delta})$, 18-nm $(\boldsymbol{\nabla}), 25-\mathrm{nm}$ $(\bowtie)$, and 30-nm (ם) coatings (Eq. 3a). (b) Layer density relative to that of pure oleic acid for increasing normalized ozone exposure, as calculated by two independent methods (Eqs. 3a and 3b). Also shown is the percent difference between the layer density calculated by Eq. (3a) versus by Eq. (3b), $\% \delta \rho_{L}$. Key: predicted $\rho_{L}$ (Eq. 3a) (ם) and predicted $\rho_{L}$ (Eq. 3b) ( $\square$ ). (c) Correlation of the layer density (Eq. 3a) with the carbonnormalized oxygen content $(z / x)$ of the average chemical composition $\mathrm{C}_{\mathrm{x}} \mathrm{H}_{\mathrm{y}} \mathrm{O}_{\mathrm{z}}$ of the reacted particles. Conditions (b) and (c): initial 30-nm oleic acid coating on polystyrene latex cores.

initial layer thickness, $d_{m}$ decreases while $d_{v a}$ increases for increasing ozone exposure.

These observations of a decrease in $d_{m}$ and an increase in $d_{v a}$ are in good agreement with related previous reports on the reaction of oleic acid aerosol particles with ozone.
Upon ozone exposure, Morris et al. (2002) and Smith et al. (2002) both report that the aerodynamic diameter of oleic acid aerosol particles increases. Broekhuizen et al. (2005) ${ }^{1}$ report that the mobility diameter decreases. More specifically, a fractional aerodynamic diameter increase of 1.02 is 
observed by Morris et al. (2002) for $600 \mathrm{~nm}$ particles for a normalized ozone exposure approximately 0.4. In comparison, an increase of 1.03 is measured in our study for 154-nm aerodynamic particles after a normalized ozone exposure of 0.6. Broekhuizen et al. (2005) ${ }^{1}$ report that the mobility diameter decreases by an amount equivalent to $25 \%$ of the particle volume after stoichiometric reaction of oleic acid with ozone, a result which is consistent with the $25 \%$ yield of nonanal in the gas-phase. This result is consistent with the $25 \%$ decrease in layer mass and layer thickness observed in our experiments at 1.0 normalized ozone exposure.

The general finding that the mobility diameter decreases while the vacuum aerodynamic diameter increases implies, for spherical particles, that particle density increases with increasing ozone exposure (cf. $\rho_{p}=\rho_{0} \chi_{a} \chi_{v}\left(d_{v a} / d_{m}\right)$ in Table 2). Regardless of initial layer thickness, layer density increases with increasing normalized ozone exposure (Eq. 3a; Fig. 5a). Overall, layer density increases from $0.89 \mathrm{~g} \cdot \mathrm{cm}^{-3}$ for pure oleic acid to $1.12 \mathrm{~g} \cdot \mathrm{cm}^{-3}$ for reacted particles at higher ozone exposures. This result confirms the earlier suggestion by Katrib et al. (2004) that layer density increases. This finding of a layer density of $1.12 \mathrm{~g} \cdot \mathrm{cm}^{-3}$ can be compared to the result of $1.09 \mathrm{~g} \cdot \mathrm{cm}^{-3}$ reported in the recent study of Broekhuizen et al. (2005) $)^{1}$. Broekhuizen et al. (2005) ${ }^{1}$ indirectly infer density from measurements of the products, their yield, and their evaporation.

The second method for calculating particle and layer densities (Eqs. 2b and 3b) is based upon measurements of layer mass and particle mobility diameter. An example of the decrease in mobility diameter with increasing ozone exposure is shown in Fig. 4 a for a particle having a 30-nm coating. The corresponding decrease in layer mass with increasing ozone exposure is shown in the aerosol mass measurements of the AMS (solid symbols in Figs. 4b) and the measurements of layer mass given both by Eqs. (1a) and (1b) (solid and open symbols, respectively, in Fig. 4c). Layer density calculated via Eq. (3b) based upon the measurements of layer mass (Eq. 1a) and the mobility diameter is shown as open symbols in Fig. 5b for increasing ozone exposure.

The two independent methods of calculating layer density agree well (Fig. 5b). The method based upon mobility and aerodynamic diameters (Eq. 3a) is systematically approximately $1.6 \%$ below the method based upon mobility diameter and particle layer mass (Eq. 3b). Uncertainties that possibly explain the systematic differences are analyzed further in the appendix.

Figure 5 shows that the particle properties change most rapidly at low ozone exposures (e.g., below 1.0) and approach limiting values at higher ozone exposures (e.g., above 3.0). This observation is consistent with the rapid reaction of ozone with oleic acid because oleic acid is, by definition, present at high concentrations for low ozone exposures. Particle properties, however, clearly continue to evolve even when oleic acid is no longer present at ozone exposures above 1.0. The implication is that the oxidation products of oleic acid with ozone continue to react with ozone (albeit at a lower reaction rate) and/or themselves. This result is consistent with the findings of Broekhuizen et al. (2005) ${ }^{1}$, who observed that the CCN properties of ozone-processed oleic acid particles continue to evolve, even up to a normalized ozone exposure of 1000.

The chemical basis for the increase in layer density is the addition of oxygen to a hydrocarbon. The atomic weight of oxygen is greater than that of either carbon or hydrogen, so the addition of oxygen to a hydrocarbon usually has the effect of increasing density. The increase in layer density is most rapid at low ozone exposures (Fig. 5b), which is consistent with the initial rapid oxygen uptake due to the formation of oxygenated products by the fast reaction of ozone with oleic acid. For example, Katrib et al. (2004) report that 9-oxononanoic acid, which is more oxygenated than oleic acid, forms with 20 to $35 \%$ carbon-normalized yield. Also reported is the formation of other, unidentified oxygenated molecules at a yield of $35-50 \%$. (Volatile products, such as 1-nonanal, which do not contribute to the layer mass, are formed at approximately 25\% yield.) Although all of the condensed-phase products cannot be identified, the overall carbon-normalized oxygen content $(z / x)$ of the $\mathrm{C}_{\mathrm{x}} \mathrm{H}_{\mathrm{y}} \mathrm{O}_{\mathrm{z}}$ organic layer can, nevertheless, be assayed by analysis of the mass spectra (cf. Katrib et al., 2004). Infrared observations by Asad et al. (2004) also indicate the formation of oxygenated functional groups and, therefore, an increase in $z / x$. Figure $5 \mathrm{c}$ shows that, as $z / x$ increases from 0.1 for unreacted oleic acid to 0.25 after high ozone exposure, layer density concomitantly increases. The relationship between layer density and $z / x$ is monotonic, though not linear. The chemical observations made by the AMS of increasing oxygen content in the chemistry of the organic layer are consistent with the physical changes apparent in the increasing layer density.

\subsection{Uncertainty analysis}

Although Table 3 shows excellent agreement among the quantities $\bar{m}_{L}, \rho_{p}$, and $\rho_{L}$ when calculated by two independent methods and thus generally validates our experimental approach, we can, nevertheless, consider several random uncertainties in our measurements and systematic errors in our analysis, which can serve to focus our future efforts to further improve measurements and calculations. The random uncertainties in our analysis derive from the precision of the primary measurements $\chi, d_{m}, d_{v a}, m_{L}$, and $N$. The systematic errors in our analysis include (1) a monodispersebased analysis for a weakly polydisperse aerosol (geometric standard deviation of 1.1), (2) an assumption of equivalency between the AMS-derived mass median diameter (MMD) and the SMPS-derived count median diameter (CMD), (3) the accuracy of $d_{m}$ and $d_{v a}$ when the SMPS and AMS are calibrated with slightly nonspherical PSL particles, (4) the accuracy of $m_{L}$ when the measured aerosol mass includes 
some organic mass in the nanoparticle mode, and (5) the accuracy of $m_{L}$ when the ionization efficiencies of the ozonolysis products differ from that of oleic acid. There are also possible effects of covarying systematic errors of $d_{m}$ and $N$ with $m_{L}$ because of the AMS calibration procedure. These random and systematic uncertainties are addressed in the appendix by beginning with an assessment of measurement uncertainties and propagating the uncertainties through the governing equations (Eqs. 1 to 3 ).

An a posteori assessment of uncertainties is also possible because we have two independent equations (i.e., Eq. 1a versus $1 b, 2 b$ versus $2 a$, or $3 a$ versus $3 b$ ). Figure 6 shows the results of aerosol mass measurements by Eq. (1a) compared to those by Eq. (1b). In Fig. 6a, the 1:1 line of aerosol mass is shown for increasing ozone exposure. The fit to the data has a slope of 1.018 , suggesting a small systematic difference with increasing ozone exposure. Random uncertainties, given by the standard deviation of the residual of data around the line of slope 1.018 , are $3 \%$. We regard these percent differences as small, and their possible sources are addressed in the appendix.

A second a posteori assessment is given in Fig. $6 \mathrm{~b}$ by comparing aerosol layer mass measured by three independent methods for unreacted layers. Aerosol layer mass can be directly measured by the AMS, can be predicted by combining Eqs. (1a) and (1b), and, for layers of unreacted oleic acid, can be predicted by Eq. (5):

$m_{L}=N\left(0.468 \mathrm{~g} / \mathrm{cm}^{3}\right)\left(d_{m}^{3} / \chi_{a}^{3}-d_{\text {core }}^{3}\right)$

This equation is obtained by substitution of Eq. (T1.4) into Eq. (1a) using the layer density of oleic acid. The comparison of aerosol layer mass obtained by these three independent methods is shown in Fig. 6b. The good agreement among these methods supports the validity of the experimental results.

\section{Conclusions}

This study introduces an innovative experimental setup that allows for multifaceted characterization of changes in the density, the chemical composition, and the shape of aerosol particles due to heterogeneous chemical reactions. The reactions of oleic acid core-shell aerosol particles with ozone are employed as a model system to illustrate the complex, nonlinear particle aging processes that are an integral part of aerosol heterogeneous chemistry. Ozone exposure is shown to decrease the mobility diameter while increasing the vacuum aerodynamic diameter, a result which implies that particle density increases. The evolution of the particle density is confirmed by two independent methods, which agree within $2 \%$. Analysis of the particle chemical composition shows that the oxygen content of the reacted organic layer increases as density increases. These changes continue even after all of the oleic acid has reacted, which indicates that chemical
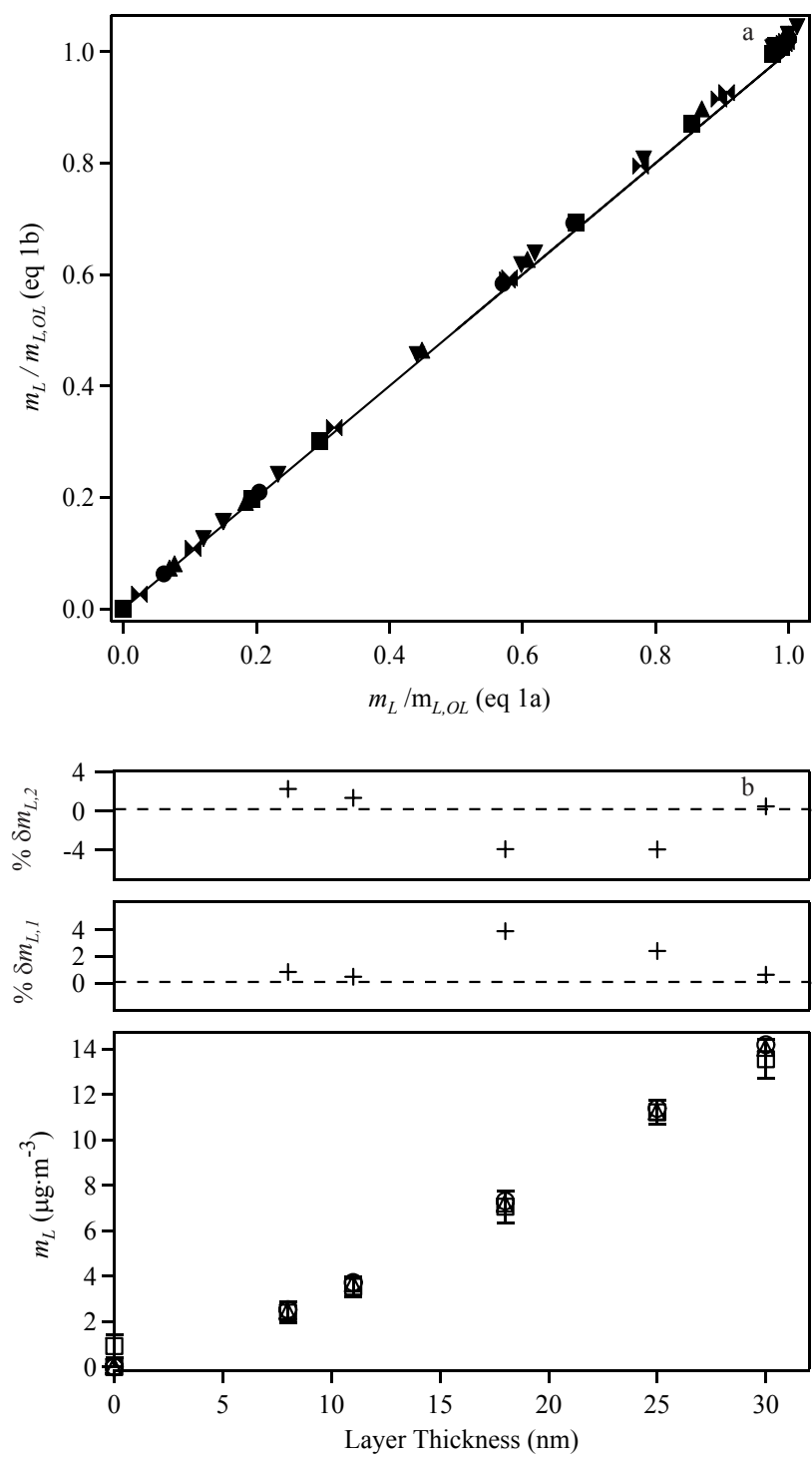

Fig. 6. (a) Comparison of $m_{L}$ predicted by Eq. (1b) versus (1a). The 1:1 line is shown. The masses are normalized to the corresponding unreacted layer mass of oleic acid. Data are shown for 8-nm $(\bullet), 11-$ $\mathrm{nm}(\boldsymbol{\Lambda})$, 18-nm ( $)$, 25-nm ( $\bowtie)$, and 30-nm (ם) coatings. (b) (btm) Three approaches for measuring layer mass for aerosol particles having oleic acid coatings of several layer thickness on polystyrene latex cores. Key: measured $m_{L}(\square), m_{L}$ predicted by combining Eqs. (1a) and (1b) (o), and $m_{L}$ predicted by Eq. (5) ( $\Delta$ ). (middle) Percent difference between the measured and predicted masses $(\square$ versus o), $\% \delta m_{L, 1}$. (top) Percent difference between the measured and predicted masses ( $\square$ versus $\Delta$ ), $\% \delta m_{L, 2}$. (The $m_{L}$ value shown at " $0 \mathrm{~nm}$ " corresponds to a layer thinner than $2 \mathrm{~nm}$, which is too thin for the SMPS but has sufficient mass for detection by the AMS.)

reactions continue, either with ozone or with themselves, for the organic products.

Aerosol heterogeneous chemistry, both in the atmosphere and in the laboratory, significantly alters the 
physical properties and the chemical composition of particles (Rudich, 2003). The evolution of particle density directly affects the mechanical, chemical, and optical properties of aerosol particles. Particle density affects aerosol removal processes and hence lifetime in the atmosphere because it directly alters aerodynamic diameter and thus the rate of dry deposition. Moreover, the changes in refractive index with density may alter the magnitude of aerosol direct radiative forcing. Particle density also impacts the deposition of inhaled particulate matter, both in its retained quantity and in its deposition locations in the lungs.

By forming polar functional groups, aerosol heterogeneous chemistry can influence particle hygroscopicity. Asad et al. (2004) have shown, for example, that increased water uptake occurs when an oleic acid film is transformed into products by reaction with ozone. Higher water uptake could possibly lead both to enhanced CCN activity and thus changes in the physical and optical properties of clouds and to an increased wet deposition rate and thus reduced atmospheric lifetime of aerosol particles.

The aerosol processes described in this paper are applicable to a wide range of olefinic organic molecules, of which oleic acid is just one member, and to a range of atmospheric oxidants, including not just ozone but also hydroxy and nitrate radicals. The approaches introduced in this paper for the study of aerosol heterogeneous chemistry will allow the continued development of more detailed and accurate aerosol process-descriptions in models of air quality and atmospheric chemistry.

\section{List of terms}

$d_{e}$ : volume equivalent diameter $(\mathrm{nm})$

$d_{m}$ : mobility diameter $(\mathrm{nm})$

$d_{v a}:$ vacuum aerodynamic diameter $(\mathrm{nm})$

$L$ : layer thickness $(\mathrm{nm})$

$m_{L}$ : aerosol layer mass $\left(\mu \mathrm{g} \cdot \mathrm{m}^{-3}\right)$ (sum of layer mass of all particles)

$\bar{m}_{L}$ : particle layer mass $\left(\mathrm{g} \cdot\right.$ particle $^{-1}$ ) (layer mass of individual particle)

$K n$ : Knudsen number $N$ : particle number concentration $\left(\# \cdot \mathrm{cm}^{-3}\right)$ measured by SMPS analysis

$\rho_{L}:$ layer density $\left(\mathrm{g} \cdot \mathrm{cm}^{-3}\right)$

$\rho_{p}:$ particle density $\left(\mathrm{g} \cdot \mathrm{cm}^{-3}\right)$

$\rho_{0}$ : unit density $\left(1.000 \mathrm{~g} \cdot \mathrm{cm}^{-3}\right)$

$\chi$ : dynamic shape factor

$\chi_{a}: \chi_{a}^{\prime}$ lumped with transition-regime Cunningham slip correction factors (see Eq. T 1.2)

$\chi_{a}^{\prime}$ : dynamic shape factor at $1 \mathrm{~atm}$ and $0.1<K n<10$ (i.e., submicron particles in the DMA)

$\chi_{v}$ : dynamic shape factor for $K n>10$ (i.e., submicron particles in the vacuum of the AMS)

\section{Appendix}

A1. Precision of the measurements and the effects of random errors

The precisions of the primary measurements of $\chi, d_{m}, d_{v a}$, $m_{L}$, and $N$, which are summarized in Table 3 , are estimated as follows. The measurement of the dynamic shape factor is taken as completely precise and accurate (i.e., $0 \%$ uncertainty) because of the evidence we have for spherical particles. The precisions of the measurements of the mobility and vacuum aerodynamic diameters are taken as $1 \%$ based upon evaluations of instrument performance (Jayne et al., 2000).

Based upon the residuals shown in Fig. 6b, we estimate that the one-sigma precision of our measurement of $m_{L}$ is $2 \%$. A related conclusion is that, under our experimental conditions, the oleic acid mass present in the particle coatings completely vaporizes at an AMS heater temperature of $350^{\circ} \mathrm{C}$ and is efficiently collected and measured by the AMS. Moreover, Eqs. (1a) and (5) are equivalent if the AMS instrument is stable from the time of calibration to the time of measurement, if the AMS signal scales linearly with mass, and if the AMS signal is independent of particle geometry (i.e., homogeneous calibration particles versus core-shell test particles). The good agreement shown in Fig. $6 \mathrm{~b}$ is a validation of these assumptions.

In our experimental setup, accuracy and precision in the measurement of the particle number density are most difficult (Ankilov et al., 2002). Although the manufacturer's manual suggests an error of $0.5 \%$ for the particle concentrations and the flow rates of the SMPS setup, our application involves integrating $\mathrm{d} N / \mathrm{d} \log d_{m}$ across the super 100-nm mode. This mode overlaps weakly with a nanoparticle mode centered around 50 to $90 \mathrm{~nm}$. Given the mode overlap, the charge correction factors, and the uncertainties in the DMA transmission function, we estimate an accuracy of 5\% in our measurement of $N$.

The uncertainties in the calculated quantities $L, \bar{m}_{L}, \rho_{p}$, and $\rho_{L}$, which are based upon the combined random uncertainties of the primary quantities, are shown in Table 3 for all layer thickness. The one-sigma uncertainties are obtained using a Monte Carlo simulation of 10000 trials. In this simulation, an equation (e.g., Eq. 2b) is evaluated repeatedly with a random variation of the input quantities within their statistical uncertainty. The mean and the standard deviation of the resulting set of numbers are the entries for the derived quantities in Table 3.

Except for $L$, the derived quantities have two independent equations for their evaluation. When the random uncertainty estimates are correct, one would expect that the calculations by the independent methods would agree with each other within experimental uncertainty. The comparison of any two columns (e.g., $\bar{m}_{L}$ by Eq. 1a versus by Eq. 1b) shows that not only is this condition met but also appears to be met even better than would be expected from the uncertainties. 
Table 4. Sensitivity analysis for experiments \#2 and \#6. The percent perturbation to the derived quantities is shown for $1 \%$ perturbation to the primary measurements.

\begin{tabular}{|c|c|c|c|c|c|c|c|c|c|c|}
\hline & & & & \multicolumn{7}{|c|}{ Sensitivity $(\% \delta)$} \\
\hline \multicolumn{3}{|c|}{ Experiment } & Perturbation & $L$ & $\bar{m}_{L}$ & $\bar{m}_{L}$ & $\rho_{p}$ & $\rho_{p}$ & $\rho_{L}$ & $\rho_{L}$ \\
\hline \multirow[t]{5}{*}{$\# 2$} & $\chi$ & 1.00 & $\delta \chi=+1 \%$ & -7.24 & 0.00 & -3.02 & 2.01 & 3.03 & 5.69 & 8.99 \\
\hline & $d_{m}$ & 117 & $\delta d_{m}=+1 \%$ & 7.31 & 0.00 & 6.13 & -0.99 & -2.94 & -2.18 & -7.83 \\
\hline & $d_{v a}$ & 118 & $\delta d_{v a}=+1 \%$ & 0.00 & 0.00 & 3.05 & 1.00 & 0.00 & 3.05 & 0.00 \\
\hline & $m_{L}$ & 2.5 & $\delta m_{L}=+1 \%$ & 0.00 & 1.00 & 0.00 & 0.00 & 0.33 & 0.00 & 1.00 \\
\hline & $N$ & 9100 & $\delta N=+1 \%$ & 0.00 & -0.99 & 0.00 & 0.00 & -0.32 & 0.00 & -0.99 \\
\hline \multirow[t]{6}{*}{ \#6 } & $\chi$ & 1.00 & $\delta \chi=+1 \%$ & -2.66 & 0.00 & -1.37 & 2.01 & 3.03 & 2.64 & 4.06 \\
\hline & $d_{m}$ & 161 & $\delta d_{m}=+1 \%$ & 2.68 & 0.00 & 2.77 & -0.99 & -2.94 & -1.20 & -3.87 \\
\hline & $d_{v a}$ & 152 & $\delta d_{v a}=+1 \%$ & 0.00 & 0.00 & 1.38 & 1.00 & 0.00 & 1.38 & 0.00 \\
\hline & $m_{L}$ & 14.1 & $\delta m_{L}=+1 \%$ & 0.00 & 1.00 & 0.00 & 0.00 & 0.72 & 0.00 & 1.00 \\
\hline & $N$ & 9500 & $\delta N=+1 \%$ & 0.00 & -0.99 & 0.00 & 0.00 & -0.72 & 0.00 & -0.99 \\
\hline & & & & Eq. (T1.5) & Eq. (1a) & Eq. (1b) & Eq. (2a) & Eq. (2b) & Eq. (3a) & Eq. (3b) \\
\hline
\end{tabular}

A bootstrap data analysis based upon comparison of the two columns of data would suggest smaller uncertainties. A reasonable conclusion is, therefore, that the uncertainty estimates given in Table 3 for the primary quantities are too large.

Convolution of the precisions of the primary measurements with the sensitivities of the derived quantities, which are respectively given in Tables 3 and 4, immediately reveals the primary measurement most affecting the random uncertainty in the calculated quantity. For example, a $5 \%$ increase in $N$ (Table 3 ) yields a $3.60 \%$ decrease (i.e., $(-0.72) /(1 \%) \times(5 \%)$; Table 4$)$ in $\rho_{p}$ calculated by Eq. (2b) for a 30-nm coating. An overall analysis by this approach shows that the random uncertainty in $L$ for a $30-n m$ coating is due mostly to uncertainty in $d_{m}$, in $\bar{m}_{L}^{\text {1a }}$ (i.e., $\bar{m}_{L}$ calculated by Eq. 1a) to $N$, in $\bar{m}_{L}^{1 \mathrm{~b}}$ to $d_{m}$, in $\rho_{p}^{2 \mathrm{a}}$ equally to $d_{m}$ and $d_{v a}$, in $\rho_{p}^{2 \mathrm{~b}}$ to $N$, in $\rho_{L}^{3 \mathrm{a}}$ to $d_{v a}$, and in $\rho_{L}^{3 \mathrm{~b}}$ to $N$. The same results hold for an 8-nm coating, except that the largest uncertainties in $\rho_{p}^{2 \mathrm{~b}}$ and $\rho_{L}^{3 \mathrm{~b}}$ derive from uncertainty in $d_{m}$ instead of in $N$. In all cases, the sensitivity of the thin layer is either equal to or greater than that of the thick layer.

The sensitivity analysis given in Table 4 shows how a $1 \%$ perturbation in any of the primary measurements $\left(\chi, d_{m}, d_{v a}\right.$, $m_{L}$, and $\left.N\right)$ affects the calculated quantities $L, \bar{m}_{L}, \rho_{p}$, and $\rho_{L}$ for thin $(8 \mathrm{~nm})$ and thick $(30 \mathrm{~nm})$ oleic acid coatings. Table 4 has great utility for assessing systematic errors in the analysis, as described in the next six sections.

A2. Monodisperse-based analysis of a weakly polydisperse aerosol

The systematic error introduced by a monodisperse-based analysis of a weakly polydisperse aerosol can be estimated by comparing the results obtained for a monodisperse dis- tribution to those obtained using a 3-bin polydisperse distribution. Based upon a geometric standard deviation (gsd) of 1.08 , the bins are centered at $\left\{d_{m} / 1.08, d_{m}, 1.08 d_{m}\right\}$ and $\left\{d_{v a} / 1.08, d_{v a}, 1.08 d_{v a}\right\}$. Each bin has a 101-nm PSL core. The particle number density in the bins is taken in the ratio 1:3:1. The mass of a layer $m_{L}$ is distributed within the bins in proportion to the layer volume. A Monte Carlo simulation to account for random uncertainties is applied. The average, mass-weighted calculated quantities $L, \bar{m}_{L}, \rho_{p}$, and $\rho_{L}$ are obtained and compared to those same quantities under the assumption of a monodisperse distribution. The results for 8- and 30-nm coatings are summarized in Table 5 under the perturbation labeled "polydispersity". The quantities most strongly affected are $\bar{m}_{L}^{1 \mathrm{~b}}, \rho_{p}^{2 \mathrm{~b}}$, and $\rho_{L}^{3 \mathrm{~b}}$. In all cases, the effects on thin layers are equal to or greater than the effects on thick layers.

A3. AMS-derived mass median diameter and the SMPSderived count median diameter

Our analysis assumes an equivalency between the AMSderived mass median diameter and the SMPS-derived count median diameter. Namely, in our analysis we obtain $d_{m}$ from the maximum of $\mathrm{d} N / \mathrm{d} \log d_{m}$ measured by the SMPS system and $d_{v a}$ from the maximum of $\mathrm{d} m_{L} / \mathrm{d} \log d_{v a}$ measured by the AMS. The Hatch-Choate conversion between CMD and MMD yields (Hinds, 1999):

$\mathrm{MMD} / \mathrm{CMD}=\exp \left(3 \ln ^{2} g s d\right)$

The ratio MMD/CMD is 1.018 for a gsd of 1.08 . The effects of a systematic reduction of $d_{v a}$ by $1.8 \%$ are shown in Table 5. The most affected quantities are $\bar{m}_{L}^{1 \mathrm{~b}}, \rho_{p}^{2 \mathrm{a}}$, and $\rho_{L}^{3 \mathrm{a}}$ for both $8-\mathrm{nm}$ and 30-nm coating. 
Table 5. Effect of systematic errors on calculated quantities. This table is derived by assuming that the perturbations given in Table 4 are linear (e.g., $\% \delta_{L}$ for $\delta d_{m}=+2 \%$ is assumed to equal $2 \% \delta_{L}$ for $\delta d_{m}=+1 \%$ ). The maximum effect of uncertainties in the ionization efficiency depends on ozone exposure and is evaluated in the table for 1.0 normalized ozone exposure (see text).

\begin{tabular}{|c|c|c|c|c|c|c|c|c|c|c|}
\hline & & & & \multicolumn{7}{|c|}{ Sensitivity $(\% \delta)$} \\
\hline \multicolumn{3}{|c|}{ Experiment } & \multirow{2}{*}{$\begin{array}{l}\text { Correction Applied } \\
\text { 1. polydispersity }\end{array}$} & \multirow{2}{*}{$\frac{\boldsymbol{L}}{1.3}$} & \multirow{2}{*}{$\frac{\overline{\boldsymbol{m}}_{\boldsymbol{L}}}{-1.6}$} & \multirow{2}{*}{$\begin{array}{c}\overline{\boldsymbol{m}}_{\boldsymbol{L}} \\
4.0\end{array}$} & \multirow{2}{*}{$\begin{array}{l}\rho_{p} \\
0.0\end{array}$} & \multirow{2}{*}{$\begin{array}{c}\rho_{p} \\
-1.6\end{array}$} & \multirow{2}{*}{$\begin{array}{l}\rho_{L} \\
0.4\end{array}$} & \multirow{2}{*}{$\begin{array}{c}\rho_{L} \\
-4.2\end{array}$} \\
\hline$\# 2$ & $\chi$ & 1.00 & & & & & & & & \\
\hline & $d_{m}$ & 117 & 2. MMD vs CMD & 0.0 & 0.0 & -2.5 & -1.8 & 0.0 & -2.5 & 0.0 \\
\hline & $d_{v a}$ & 118 & 3. PSL calibration & -7.3 & 0.0 & -3.1 & 2.0 & 2.9 & 5.2 & 7.8 \\
\hline & $m_{L}$ & 2.5 & 4. nanomode mass & 0.0 & -2.5 & 0.0 & 0.0 & -0.8 & 0.0 & -2.5 \\
\hline & $N$ & 9100 & 5. ionization efficiency & 0.0 & -1.7 & 0.0 & 0.0 & -0.6 & 0.0 & -1.7 \\
\hline \multirow[t]{6}{*}{ \#6 } & $\chi$ & 1.00 & 1. polydispersity & 0.5 & -0.1 & 2.6 & 0.0 & -1.6 & 0.1 & -2.2 \\
\hline & $\hat{d}_{m}$ & 161 & 2. MMD vs CMD & 0.0 & 0.0 & -5.4 & -1.8 & 0.0 & -5.4 & 0.0 \\
\hline & $d_{v a}$ & 152 & 3. PSL calibration & -2.7 & 0.0 & -1.4 & 2.0 & 2.9 & 2.6 & 3.9 \\
\hline & $m_{L}$ & 14.1 & 4. nanomode mass & 0.0 & -2.5 & 0.0 & 0.0 & -1.8 & 0.0 & -2.5 \\
\hline & $N$ & 9500 & 5. ionization efficiency & 0.0 & -1.7 & 0.0 & 0.0 & -1.2 & 0.0 & -1.7 \\
\hline & & & & Eq. (T1.5) & Eq. (1a) & Eq. (1b) & Eq. (2a) & Eq. (2b) & Eq. (3a) & Eq. $(3 b)$ \\
\hline
\end{tabular}

Although this analysis suggests that routinely accounting for the entire size distribution in our calculations would lead to more accurate results, there are complicating factors due to the tail in the data of the vacuum aerodynamic diameter (Fig. 3a). The tail in the size distribution measurements of the AMS and its change with increasing ozone exposure (Fig. 3a), both of which are absent in the corresponding SMPS data (Fig. 3b), arise from the low volatility of oleic acid and the further reduced volatility of the reaction products. Namely, because the AMS measurement is based upon time of flight, a slower vaporization of reacted particles as they strike the vaporizing heater explains the tail in the AMS data. The extension of the tail following ozone exposure is consistent with the decreased volatility of the reaction products compared to the parent material of oleic acid. For this reason, we restrict the analysis to mode size, which allows us to correlate particles measured by the AMS with those measured by the SMPS, to the extent that the MMD and CMD are the same.

\section{A4. Nonspherical PSL calibration particles}

The calibration of $d_{m}$ and $d_{v a}$ in the SMPS and AMS, respectively, assumes spherical particles, although our measurement of the beam profile demonstrates that uncoated PSL particles are slightly nonspherical. An approximate estimate of $\chi=1.01$ for calibration PSL particles can be made by comparing the PSL beam profile to that of liquid particles (Fig. 2b). In this case, the measured $d_{m}$ is systematically underreported by $1 \%$. Similarly, the measured $d_{v a}$ is systematically overreported by $1 \%$. Table 5 summarizes the effects of $\delta d_{m}=-1 \%$ and $\delta d_{v a}=+1 \%$ on the calculated quantities for 8 - and $30-\mathrm{nm}$ coatings. The changes for the $30-\mathrm{nm}$ coating are less than or equal to those of the 8-nm coating.

\section{A5. Nanomode mass}

The measured $m_{L}$ should be reduced to correct for the organic mass in the 50- to 90-nm nanoparticle mode. Unlike layer thickness, the mass in the nanomode is not highly reproducible in the experiments. However, 5\% is an upper limit of the observations. Although the absolute mass in the nanomode decreases for thinner layer thickness, the relative mass remains roughly constant. The effects of a systematic reduction of $m_{L}$ by $2.5 \%$ are shown in Table 5 . Most affected are $\bar{m}_{L}^{1 \mathrm{a}}$ and $\rho_{L}^{3 \mathrm{~b}}$, which are both reduced by $2.5 \%$.

\section{A6. Ionization efficiencies of ozonolysis products}

The MS signal intensity of a semi-volatile species is proportional to its ionization efficiency (Jayne et al., 2000). Therefore, any error in the employed ionization efficiency leads to an error in $m_{L}$. We apply the calibration for oleic acid to all organic molecules, thus assuming the ionization efficiency is invariant throughout the molecular family of oleic acid and its ozonolysis products (Katrib et al., 2004). Katrib et al. (2004) show that the known products, including azelaic acid, nonanoic acid, and 9-oxononaoic acid, do have comparable ionization efficiencies as oleic acid. However, this assumption cannot be tested for other ozonlysis products due to their unknown chemical structure and hence absence of calibration compounds. The uncertainty in $m_{L}$ therefore increases with ozone exposure due to the loss of oleic acid and the formation of some products of unknown ionization efficiency.

The maximum impact of this assumption can be estimated by assigning all deviation from the 1:1 line of Fig. 6a to a systematic variation in ionization efficiency. Under this treatment, Eq. (1b) is taken as totally accurate, and the deviation 
Table 6. Effect of systematic errors of $d_{m}$ or $N$ on calculated quantities when including the effect of a covarying systematic error in the AMS calibration. "PSL calibration" corresponds to $\delta d_{m}=+1 \%, \delta m_{L}=+3 \%$, and $\delta d_{v a}=-1 \%$.

\begin{tabular}{|c|c|c|c|c|c|c|c|c|c|c|}
\hline & & & & \multicolumn{7}{|c|}{ Sensitivity $(\% \delta)$} \\
\hline \multicolumn{3}{|c|}{ Experiment } & Covariance Perturbation & $L$ & $\bar{m}_{L}$ & $\bar{m}_{L}$ & $\rho_{p}$ & $\rho_{p}$ & $\rho_{L}$ & $\rho_{L}$ \\
\hline \multirow[t]{5}{*}{$\# 2$} & $\chi$ & 1.00 & $\delta d_{m}=+1 \% \rightarrow \delta m_{L}=+3 \%$ & 7.31 & 3.00 & 6.13 & -0.99 & -1.95 & -2.18 & -4.83 \\
\hline & $d_{m}$ & 117 & $\delta N=+1 \% \rightarrow \delta m_{L}=+1 \%$ & 0.00 & 0.00 & 0.00 & 0.00 & 0.00 & 0.00 & 0.00 \\
\hline & $d_{v a}$ & 118 & PSL calibration (see caption) & -7.3 & -3.0 & -3.1 & 2.0 & 2.0 & 5.2 & 4.8 \\
\hline & $m_{L}$ & 2.5 & & & & & & & & \\
\hline & $N$ & 9100 & & & & & & & & \\
\hline \multirow[t]{6}{*}{ \#6 } & $\chi$ & 1.00 & $\delta d_{m}=+1 \% \rightarrow \delta m_{L}=+3 \%$ & 2.68 & 3.00 & 2.77 & -0.99 & -0.78 & -1.20 & -0.87 \\
\hline & $d_{m}$ & 161 & $\delta N=+1 \% \rightarrow \delta m_{L}=+1 \%$ & 0.00 & 0.00 & 0.00 & 0.00 & 0.00 & 0.00 & 0.00 \\
\hline & $d_{v a}$ & 152 & PSL calibration (see caption) & -2.7 & -3.0 & -1.4 & 2.0 & 0.8 & 2.6 & 0.9 \\
\hline & $m_{L}$ & 14.1 & & & & & & & & \\
\hline & $N$ & 9500 & & & & & & & & \\
\hline & & & & Eq. (T1.5) & Eq. (1a) & Eq. (1b) & Eq. (2a) & Eq. (2b) & Eq. (3a) & Eq. (3b) \\
\hline
\end{tabular}

between Eqs. (1a) and (1b) provides an estimate of the variation of the ionization efficiency. The slope of the comparison line is 1.018, implying that the recorded mass of the ozonolysis products is slightly too high and, therefore, that the ionization efficiency of the ozonolysis products is slightly less than that of oleic acid. The impact is estimated by $\left(\% \delta m_{L}\right)_{\max }=-1.8 \%\left(1-m_{L} / m_{L, O L}\right)$. The equation shows that the maximum error increases with loss of oleic acid. Table 5 shows the effect for $\left(\% \delta m_{L}\right)_{\max }=-1.7 \%$, which corresponds to 1.0 normalized ozone exposure. Most affected are $\bar{m}_{L}^{1 \mathrm{a}}$ and $\rho_{L}^{3 \mathrm{~b}}$.

\section{A7. Covariance of errors in $m_{L}$ with errors in $N$ and $d_{m}$}

The calibration of the AMS signal intensity to aerosol mass is based upon the SMPS measurements of a monodisperse test aerosol of homogeneous particles. Specifically, the aerosol mass of size-classified spherical 350-nm oleic acid particles (density of $0.895 \mathrm{~g} \cdot \mathrm{cm}^{-3}$ ) is calculated using the measured $N$. This aerosol mass is the primary standard for the calibration of the AMS signal intensity to the oleic acid aerosol mass. Therefore, any systematic errors in the accuracy of $d_{m}$ $(350 \mathrm{~nm})$ or $N(350 \mathrm{~nm})$ lead to covarying systematic errors in the accuracy of the measured mass. (Once calibrated, the mass determined via the AMS signal intensities is independent of measurements of $d_{m}$ and $N$ so that random uncertainties do not covary.)

The covariance of $\% \delta m_{L}$ with $\% \delta d_{m}$ and $\% \delta N$ is as follows:

$m_{L}=\left(I_{M S} / I_{M S}^{*}\right)\left(\frac{\pi}{6} N^{*}\left(d_{m}^{*}\right)^{3}\right)_{S M P S}$

$\delta m_{L}=\frac{\pi}{6}\left(I_{M S} / I_{M S}^{*}\right)\left(\left(d_{m}^{*}\right)^{3} \delta N^{*}+3 N^{*}\left(d_{m}^{*}\right)^{2} \delta d_{m}^{*}\right)$ $\frac{\delta m_{L}}{m_{L}}=\frac{\delta N^{*}}{N^{*}}+3 \frac{\delta d_{m}^{*}}{d_{m}^{*}}$

$\% \delta m_{L}=3\left(\% \delta d_{m}\right)+\% \delta N$

where $I_{M S}$ is the mass spectral signal intensity, the superscript $*$ designates calibration conditions, and the subscript SMPS emphasizes the technique employed to calibrate aerosol mass.

Table 6 shows the net effect of systematic errors in $d_{m}$ and $N$ on the calculated quantities, which are calculated by using the entries in Table 4 for cases of (1) $\delta d_{m}=+1 \%$ and $\delta m_{L}=+3 \%$ and (2) $\delta N=+1 \%$ and $\delta m_{L}=+1 \%$ where the relationship of $\delta m_{L}$ to $\delta d_{m}$ and $\delta N$ is established by Eq. (A2.4). Notably, Table 6 shows that the effects of a systematic error in $N$ and of a covarying error in $m_{L}$ cancel. In contrast, the covariance of $m_{L}$ has the effect of increasing the error in $\bar{m}_{L}^{1 \mathrm{a}}$ but decreasing it for $\rho_{L}^{2 \mathrm{~b}}$ and $\rho_{L}^{3 \mathrm{~b}}$ for a systematic error in $d_{m}$. Therefore, the "PSL calibration" correction is also affected, and the revised values are given in Table 6.

(A comment of caution is necessary in the use of Eq. $2 b$ to avoid a circular measurement. Namely, if Eq. $2 b$ is applied to the study of homogeneous oleic acid particles - i.e., the calibration particles - then Eq. 2 b collapses into $\rho_{p}=\rho_{p}$, and no true measurement is made. Perturbations from the calibration system, such as $d_{\text {core }}>0$ or a change in the chemical makeup of the particle through ozone exposure, restore Eq. $2 \mathrm{~b}$ as an independent method.)

\section{A8. Conclusions}

Our analysis suggests a systematic explanation for several trends apparent in the data. Notably, regardless of layer 
thickness, the particle density of unreacted particles is consistently $1 \%$ larger than would be expected based upon geometric calculation (e.g., $0.944 \mathrm{~g} \cdot \mathrm{cm}^{-3}$ measured versus $0.934 \mathrm{~g} \cdot \mathrm{cm}^{-3}$ expected). As a result, although oleic acid has a density of $0.895 \mathrm{~g} \cdot \mathrm{cm}^{-3}$, the reported layer density of unreacted oleic acid decreases from 0.928 to $0.908 \mathrm{~g} \cdot \mathrm{cm}^{-3}$ ( $3.6 \%$ to $1.4 \%$ too large) as layer thickness increases from 8 to $30 \mathrm{~nm}$ (Table 3). Tables 5 and 6 show that layer density would be reduced if polydispersity and nanomode mass were accounted for and would be increased if the diameter and AMS calibrations with nonspherical PSL particles were considered.

The results shown in Tables 5 and 6 suggest a priority ranking for improved analysis. The highest priority is to address the issue of $d_{m}$ and $d_{v a}$ calibration with the PSL particles. A further recommendation is to calibrate the AMS by a method independent of the SMPS measurements, preferably by a method directly sensitive to aerosol mass (e.g., by infrared light absorption in the nonscattering size regime). The next priorities are to distinguish between MMD vs. CMD when emphasis is placed on thicker coatings or to treat aerosol polydispersity when emphasis is placed on thinner coatings.

Acknowledgements. We are grateful for support received from the National Science Foundation Atmospheric Chemistry Program (ATM-0215357) and the Dreyfus Foundation Postdoctoral Program in Environmental Chemistry. We thank J. Donaldson, K. Broekhuizen, and G. Smith for advance access to their manuscripts cited herein. Valuable discussion with J.-L. Jimenez is appreciated.

Edited by: M. Kulmala

\section{References}

Ankilov, A., Baklanov, A., Colhoun, M., Enderle, K. H., Gras, J., Julanov, Y., Kaller, D., Lindner, A., Lushnikov, A. A., Mavliev, R., McGovern, F., Mirme, A., O'Connor, T. C., Podzimek, J., Preining, O., Reischl, G. P., Rudolf, R., Sem, G. J., Szymanski, W. W., Tamm, E., Vrtala, A. E., Wagner, P. E., Winklmayr, and Zagaynov, V.: Intercomparison of number concentration measurements by various aerosol particle counters, Atmos. Res., 62, 177-207, 2002

Asad, A. B., Mmereki B. T., and Donaldson, D. J.: Enhanced uptake of water by oxidatively processed oleic acid, Atmos. Chem. Phys. Discuss., 4, 4019-4038, 2004,

\section{SRef-ID: 1680-7375/acpd/2004-4-4019.}

Baron, A. and Willeke, K.: Aerosol Measurement: Principles, Techniques, and Applications, New York, Wiley, 2001.

DeCarlo, P., Slowik, J. G., Worsnop, D. R., Davidovits, P., and Jimenez, J. L.: Particle morphology and density characterization by combined mobility and aerodynamic diameter measurements, Part 1: Theory, Aerosol Sci. Technol., 12, 1185-1205, 2004.

Ehara, K., Hagwood, C., and Coakley, K. J.: Novel method to classify aerosol particles according to their mass-to-charge ratio -
Aerosol particle mass analyser, J. Aerosol. Sci., 27, 217-234, 1996.

Fuchs, N. A.: The Mechanics of Aerosol, Pergamon Press, Oxford, 1964.

Hand, J. L. and Kreidenweis, S. M.: A new method for retrieving particle refractive Index and effective density from aerosol size distribution data, Aerosol Sci. Technol., 36, 1012-1026, 2002.

Hearn, J. D. and Smith, G. D.: Kinetics and products studies for ozonolysis reactions of organic particles using aerosol CIMS, J. Phys. Chem. A, 108, 10 019-10 029, 2004.

Hinds, W. C.: Aerosol Technology: Properties, Behavior, and Measurement of Airborne Particles, New York, Wiley, 1999.

Jayne, J. T., Leard, D. C., Zhang, X., Davidovits, P., Smith, K. A., Kolb, C. E., and Worsnop, D. R.: Development of an aerosol mass spectrometer for size and composition analysis of submicron particles, Aerosol. Sci. Technol., 33, 49-70, 2000.

Jimenez, J. L., Cocker, D. R., Bahreini, R., Zhuang, H., Varutbangkul, V., Flagan, R. C., Seinfeld, J. H., O’Dowd, C. D., and Hoffmann, T.: New particle formation from photooxidation of diiodomethane (CH2I2), J. Geophys. Res., 108, 4318, 2003a.

Jimenez, J. L., Jayne, J. T., Shi, Q., Kolb, C. E., Worsnop, D. R., Yourshaw, I., Seinfeld, J. H., Flagan, R. C., Zhang, X. F., Smith, K. A., Morris, J. W., and Davidovits, P.: Ambient aerosol sampling using the aerodyne aerosol mass spectrometer, J. Geophys. Res., 108 (D7), 8425, doi:10.1029/2001JD001213, 2003. 2003 b.

Katrib, Y., Martin, S. T., Hung, H. M., Rudich, Y., Zhang, H., Slowik, J. G., Davidovits, P., Jayne, J. T., and Worsnop, D. R.: Products and mechanisms of ozone reactions with oleic acid for aerosol particles having core-shell morphologies, J. Phys. Chem. A, 108, 6686-6695, 2004.

Kelly, W. P. and McMurry, P. H.: Measurement of particle density by inertial classification of differential mobility analyzer generated monodisperse aerosols, Aerosol Sci. Technol., 17, 199-212, 1992.

Lipowicz, P. J.: Determination of cigarette-smoke particle density from mass and mobility measurements in a millikan cell, J. Aerosol. Sci., 19, 587-589, 1988.

Liu, P., Ziemann, P. J., Kittelson, D. B., and McMurry, P. H.: Generating particle beams of controlled dimensions and divergence: I. Theory of particle motion in aerodynamic lenses and nozzle expansions, Aerosol Sci. Technol., 22, 293-313, 1995a.

Liu, P., Ziemann, P. J., Kittelson, D. B., and McMurry, P. H.: Generating particle beams of controlled dimensions and divergence: II. Experimental evaluation of particle motion in aerodynamic lenses and nozzle expansions, Aerosol Sci. Technol., 22, 314324, 1995b.

McMurry, P. H., Wang, X., Park, K., and Ehara, K.: The relationship between mass and mobility for atmospheric particles: A new technique for measuring particle density, Aerosol Sci. Technol., 36, 227-238, 2002.

Moise, T. and Rudich, Y.: Reactive uptake of ozone by aerosolassociated unsaturated fatty acids: Kinetics, mechanism, and products, J. Phys. Chem. A, 106, 6469-6476, 2002.

Morris, J. W., Davidovits, P., Jayne, J. T., Jiménez, J. L., Shi, Q., Kolb, C. E., Worsnop, D. R., Barney, W. S., and Cass, G.: Kinetics of submicron oleic acid aerosols with ozone: A novel aerosol mass spectrometric technique, Geophys. Res. Lett., 29, 1357, doi:10.1029/2002GL014692, 2002. 
Murphy, D. M., Cziczo, D. J., Hudson, P. K., Schein, M. E., and Thomson, D. S.: Particle density inferred from simultaneous optical and aerodynamic diameters sorted by composition, J. Aerosol. Sci., 35, 135-139, 2004.

Ristimaki, J., Virtanen, A., Marjamaki, M., Rostedt, A., and Keskinen, J.: On-line measurement of size distribution and effective density of submicron aerosol Particles, J. Aerosol. Sci., 33, 1541-1557, 2002.

Rudich, Y.: Laboratory perspectives on the chemical transformations of organic matter in atmospheric particles, Chem. Rev., 103, 5097-5124, 2003.

Seinfeld, J. H. and Pandis, S. N.: Atmospheric Chemistry and Physics, New York, Wiley, 1998.
Smith, G. D., Woods, E., DeForest, C. L., Baer, T., and Miller, R. E.: Reactive uptake of ozone by oleic acid aerosol particles: Application of single-particle mass spectrometry to heterogeneous reaction kinetics, J. Phys. Chem. A, 106, 8085-8095, 2002.

Smith, G. D., Woods III, E., Baer, T., and Miller, R. E.: Aerosol uptake described by numerical solution of the diffusion - Reaction equations in the particle, J. Phys. Chem. A, 107, 9582-9587, 2003.

Thornberry, T. and Abbatt, J. P. D.: Heterogeneous reaction of ozone with liquid unsaturated fatty acids: Detailed kinetics and gas-phase product studies, Phys. Chem. Chem. Phys., 6, 84-93, 2004.

Ziemann, P. J.: American Association for Aerosol Research (AAAR), Annual Meeting, Anaheim, 2003. 